\title{
CHROMOSOME STUDIES IN ASTERACEAE FROM THE UNITED STATES, MEXICO, THE WEST INDIES, AND SOUTH AMERICA ${ }^{1}$
}

\author{
David J. Keil, Melissa A. Luckow, and Donald J. Pinkava \\ Biological Sciences Department, California Polytechnic State University, San Luis Obispo, \\ California 93407; Department of Botany, University of Texas, Austin, Texas 78712; Department of \\ Botany and Microbiology, Arizona State University, Tempe, Arizona 85287
}

\begin{abstract}
A B S T R A C T
Chromosome counts of Asteraceae are reported from Mexico, the United States, the West Indies, Peru, and Bolivia. First counts are reported for 27 species, eight infraspecific taxa, and three interspecific hybrids in Brickellia, Chrysanthellum, Cirsium, Egletes, Erigeron, Flaveria, Gnaphalium, Heterotheca, Hieracium, Hymenothrix, Koanophyllon, Layia, Lessingia, Pectis, Sclerocarpus, Stuessya, Tagetes and Wedelia. Counts are also reported for 196 taxa or hybrids for which chromosome numbers have been published previously. Of these, nine are new numbers. Taxonomic implications of certain counts are discussed.
\end{abstract}

THIS PAPER is a continuation of studies (Keil and Stuessy, 1975, 1977; Keil and Pinkava, 1976, 1979, 1981; Keil, 1977; Pinkava and Keil, 1977) on chromosome numbers in Asteraceae. Vouchered chromosome counts represent both hitherto unreported taxa and taxa for which counts have been published previously. The former counts serve to increase the number of taxa for which cytological data are available and can provide insights into relationships and patterns of evolution. The latter counts provide information about variation in chromosome complements and broaden the geographical sample of the various taxa.

METHODS-Immature capitula were killed and fixed in modified Carnoy's fixative [4-6 chloroform : 3 ethanol : 1 glacial acetic acid, v:v: v]. Florets were stained in either iron-acetocarmine or Snow's stain (Snow, 1963) and anthers or whole florets were squashed in a drop of Hoyer's medium in a modification of the methods of Beeks (1955). Chromosome counts and meiotic behavior were ascertained from

\footnotetext{
${ }^{1}$ Received for publication 17 March 1987; revision accepted 14 July 1987.

Research was supported by NSF Grant DEB 81-04683. We thank John Popenoe, Director of the Fairchild Tropical Gardens, for making facilities available during Dr. Keil's field work in the West Indies; and George Proctor and Peter Bretting for assistance in the field in the Dominican Republic and Jamaica, respectively; Judith M. Canne for providing bud samples from Bolivian plants; Michael O. Dillon for providing seeds of Peruvian plants; and John C. Semple for determinations for Heterotheca and Pityopsis species. We particularly thank R. K. Brown for allowing us to publish results from his research on Tragopogon. We thank Billie L. Turner, John C. Semple, and John L. Strother for reviewing the manuscript.
}

meiotic microsporocytes and were documented by camera lucida or free-hand drawings. Voucher specimens, microslides, and drawings are deposited at ASU and OBI. Percent pollen stainability, based on 500 grain samples stained in aniline blue in lactophenol, was calculated for some plants.

RESULTS-The chromosome counts obtained are listed in Table 1. First counts are reported for 27 species, eight infraspecific taxa, and three interspecific hybrids; counts are also reported for 196 additional taxa or hybrids for which counts have been published previously. Eight of these are new numbers.

Discussion-As in previous papers, the discussion is restricted to first or new counts or significant comments. The sequence of Table 1 and the accompanying commentary is alphabetical by tribe.

Astereae-Our report of $2 n=9_{\text {II }}$ for Egletes prostrata and the only previous report for the genus, $n=27$ for $E$. viscosa (L.) Less. var. dissecta Shinners (Keil and Stuessy, 1977), support a base number of $x=9$ for the genus.

Our first report of $2 n=9_{\mathrm{II}}$ for Erigeron bellioides is in accord with the established base number, $x=9$, for Erigeron. The head morphology of this diminutive plant, however, suggests that it might better be placed into $\mathrm{Co}$ nyza, which has the same base number. Cronquist (1943) listed the following features as diagnostic for Conyza: "Central hermaphrodite flowers few; pistillate flowers numerous, with filiform corollas; ligules, if present, inconspicuous, shorter than the tubes and scarcely if at all exceeding the pappus." The heads 
TABLE 1. Chromosome counts of Asteraceae. Symbols: * Indicates first report for a taxon or hybrid combination. ** Indicates previously unreported chromosome count for a taxon. Abbreviations of collectors: $M G K=M a l c o l m G$. McLeod, Timothy Gaskin and David Keil; $K=$ David Keil; KB = David Keil and Peter Bretting; KL = David Keil and Melissa Luckow; KLL = David Keil, Melissa Luckow and David Luckow; KPr = David Keil and George R. Proctor; $L=$ Elinor Lehto; $M K=$ Malcolm G. McLeod and David Keil; MMK=Malcolm G. McLeod, Henry G. Meyer and David Keil; $P=$ Donald J. Pinkava

\begin{tabular}{lll}
\hline \hline $\begin{array}{l}\text { Tribe } \\
\text { Taxon or hybrid combination }\end{array}$ & Chromosome count & Location and voucher \\
\hline $\begin{array}{l}\text { ANTHEMIDEAE } \\
\text { Achillea millefolium } \mathrm{L} .\end{array}$ & $2 n=18_{\mathrm{II}}$ & $\begin{array}{l}\text { UNITED STATES: Virginia: Smyth Co.: White } \\
\text { Top Mtn, K12820 pop. (OBI) }\end{array}$ \\
Artemisia californica Less. & $2 n=9_{\mathrm{II}}$ & $\begin{array}{l}\text { UNITED STATES: California: Kern Co.: Blue- } \\
\text { stone Ridge below Orchard Peak, } M G K 11846 \\
\text { (OBI) }\end{array}$
\end{tabular}

ASTEREAE

Acamptopappus sphaerocephalus (H. \& $2 n=9_{\mathrm{II}}$ G.) A. Gray

Astranthium condimentum DeJong

$2 n=3_{\text {II }}$

Chrysopsis pilosa Nutt.

$2 n=4_{\text {II }}$

Chrysothamnus nauseosus (Pall.) Britt. $\quad 2 n=9_{\mathrm{II}}$ ssp. hololeuca (A. Gray) Hall \& Clem.

Chrysothamnus viscidiflorus (Hook.) $\quad 2 n=9_{11}$ Nutt.

Conyza canadensis (L.) Cronq.

$$
n=9
$$

Conyza filaginoides (DC.) Heiron.

Conyza aff. sophiifolia H.B.K.

Corethrogyne filaginifolia (H. \& A.)

Nutt. var. robusta Greene

*Egletes prostrata (Sw.) Ktze.

Ericameria arborescens (A. Gray)

Greene

*Erigeron bellioides DC.

Erigeron divergens T. \& G.

$$
\begin{gathered}
2 n=9_{\text {II }} \\
n=9 \\
2 n=5_{\text {II }} \\
2 n=9_{\text {II }}
\end{gathered}
$$

$2 n=9_{11}$

$2 n=9_{\text {II }}$

$2 n=3 x=27$

UNITED STATES: California: Los Angeles Co.: 5 mi W of San Bernardino Co. line on Rt 138, K11894 pop. (OBI)

MEXICO: Michoacán: Rt 37, 3 mi S of jet with Rt 15, KL15167 (OBI)

UNITED STATES: North Carolina: Cumberland Co.: Fort Bragg, K11650 (ASU)

UNITED STATES: California: Los Angeles Co.: 5 $\mathrm{mi} \mathrm{W}$ of San Bernardino Co. line on Rt 138, K11895 pop. (OBI)

UNITED STATES: Wyoming: Natrona Co.: Alcova, $K 12870$ (OBI)

UNITED STATES: Arizona: Maricopa Co.: McDowell Mtns, Lane 1737 (ASU); California: Monterey Co.: $6 \mathrm{mi}$ N of Castroville, K10945 (OBI, ASU); Montana: Carbon Co.: Devil's Canyon area, R. K. Brown 491-1 (ASU)

MEXICO: México: $4.5 \mathrm{mi}$ W of Santa María del Monte, KL15413A (OBI)

MEXICO: Durango: Rt 40, $19.5 \mathrm{mi}$ E of La Ciudad, P13399 (ASU)

UNITED STATES: California: San Luis Obispo Co.: Morro Bay, MMK11856 (OBI)

ANTIGUA AND BARBUDA: Antigua: St. George's Parish: Windthorpes Bay, K16554 pop. (OBI)

UNITED STATES: California: San Luis Obispo Co.: Cuesta Grade, K11855 pop. (OBI)

PUERTO RICO: Isla de Cabra, K16503 pop. (OBI)

UNITED STATES: Arizona: Santa Cruz Co.: Arizona Rt 289 S of Peña Blanca Lake, L20379 (ASU) [pollen stainability $23.8 \%$; macropollen and micropollen present]; Yavapai Co.: I-17, $0.5 \mathrm{mi} \mathrm{N}$ of Sunset Point, L19918 (ASU) [pollen stainability $16.4 \%$; macropollen and micropollen present]

Erigeron flagellaris A. Gray $2 n=9_{\mathrm{II}}$

*Erigeron foliosus Nutt. var. blochman- $2 n=9_{\mathrm{II}}$ iae (Greene) Hall

Erigeron formosissimus Greene var. $\quad 2 n=9_{\mathrm{II}}$ formosissimus

Erigeron platyphyllus Greene

$2 n=9_{\text {II }}$

Erigeron strigosus Muhl.

$2 n=9_{\text {II }}$

$2 n=3 x=27$

UNITED STATES: Arizona: Coconino Co.: San Francisco Peaks, K11516 (ASU)

UNITED STATES: California: San Luis Obispo Co.: Montaña de Oro State Park, MMK11861 (OBI)

UNITED STATES: Arizona: Coconino Co.: Mt. Elden, K11515 (ASU)

UNITED STATES: Arizona: Coconino Co.: San Francisco Peaks, P13718 (ASU); West Fork, Oak Creek L20218 (ASU)

UNITED STATES: Virginia: Smyth Co.: White Top Mtn K12823 (OBI)

UNITED STATES: North Carolina: Cumberland Co.: Fort Bragg, K11636 (ASU). Pollen stain- 
TABle 1. Continued

\begin{tabular}{|c|c|c|}
\hline $\begin{array}{l}\text { Tribe } \\
\text { Taxon or hybrid combination }\end{array}$ & Chromosome count & Location and voucher \\
\hline & & $\begin{array}{l}\text { ability } 25.9 \% \text {; macropollen and micropollen } \\
\text { present }\end{array}$ \\
\hline Grindelia inuloides Willd. & $2 n=66_{I I}$ & MEXICO: Jalisco: Ajijic, KL15602 (OBI) \\
\hline Grindelia procera Greene & $\begin{array}{l}2 n=10_{\mathrm{II}}+1_{\mathrm{IV}}+2 \\
\quad \text { fragments }\end{array}$ & $\begin{array}{l}\text { UNITED STATES: California: Kern Co.: Blue- } \\
\text { stone Ridge below Orchard Peak, } M G K 11842 \\
\text { (OBI) }\end{array}$ \\
\hline Gundlachia corymbosa (Urb.) Britt. & $2 n=9_{\mathrm{II}}$ & $\begin{array}{l}\text { ANTIGUA AND BARBUDA: Barbuda: along } \\
\text { sand road to Palmetto } \mathrm{Pt}, K 16562 A(\mathrm{OBI})\end{array}$ \\
\hline Gutierrezia california (DC.) T. \& G. & $2 n=8_{I I}$ & $\begin{array}{l}\text { UNITED STATES: California: Kern Co.: Keck } \\
\text { Rd, } 4.3 \mathrm{mi} \text { SE of jct with Baker Rd and Twis- } \\
\text { selman Rd, } M G K 11839(\mathrm{OBI})\end{array}$ \\
\hline Gutierrezia wrightii A. Gray & $2 n=4_{I I}$ & $\begin{array}{l}\text { UNITED STATES: ARIZONA: Santa Cruz Co.: } \\
2.7 \text { mi from Lochiel-Nogales Rd on Patagonia } \\
\text { Rd, } K 12598 A(\mathrm{OBI})\end{array}$ \\
\hline $\begin{array}{l}\text { Gymnosperma glutinosum (Spreng.) } \\
\text { Less. }\end{array}$ & $2 n=8_{I I}$ & $\begin{array}{l}\text { MEXICO: Puebla: } 14.2 \text { mi SE of Acatlán, } \\
\text { KL } 15482 \text { (OBI) }\end{array}$ \\
\hline Heterotheca echioides (Benth.) Shinners & $2 n=9_{11}$ & $\begin{array}{l}\text { UNITED STATES: California: Monterey Co.: } \\
\text { along Carmel Valley Rd } 18 \mathrm{mi} \text { NW of Arroyo } \\
\text { Seco Rd, K11949 (ASU, OBI) }\end{array}$ \\
\hline Heterotheca grandiflora Nutt. & $n=9$ & $\begin{array}{l}\text { UNITED STATES: California: Monterey Co.: } 4 \\
\text { mi N of Castroville, K10953 (ASU); San Luis } \\
\text { Obispo Co.: Hazard Canyon, Montaña de Oro } \\
\text { State Park, K10977A (ASU) }\end{array}$ \\
\hline $\begin{array}{l}\text { Heterotheca fulcrata (Greene) Shinners } \\
\text { var. fulcrata }\end{array}$ & $n=18$ & $\begin{array}{l}\text { UNITED STATES: New Mexico: Sandoval Co.: } 4 \\
\text { mi N of San Ysidro, K10882 pro parte (ASU) }\end{array}$ \\
\hline $\begin{array}{l}\text { Heterotheca aff. fulcrata (Greene) Shin- } \\
\text { ners }\end{array}$ & $n=9$ & $\begin{array}{l}\text { UNITED STATES: Arizona: Coconino Co.: } \\
\text { Grand Canyon, rd to Pt Imperial, K11715 } \\
\text { (ASU); Coconino Co.: Oak Creek Canyon, } \\
K 11760 \text { (ASU) }\end{array}$ \\
\hline Heterotheca inuloides Cass. & $2 n=9_{\mathrm{II}}$ & $\begin{array}{l}\text { MEXICO: Oaxaca: } 31.3 \mathrm{mi} \mathrm{S} \text { of Cuicatlán, } \\
\text { KL15574 pop. (OBI) }\end{array}$ \\
\hline $\begin{array}{l}\text { Heterotheca latifolia Buckl. var. latifo- } \\
\quad \text { lia }\end{array}$ & $n=9$ & $\begin{array}{l}\text { UNITED STATES: North Carolina: Cumberland } \\
\text { Co.: Ft. Bragg Military Reservation, K11609 } \\
\text { (ASU); Texas: Big Thicket area, Village Creek, } \\
\text { Somers \& Keil } 10592 \text { (ASU) }\end{array}$ \\
\hline $\begin{array}{l}\text { Heterotheca mexicana Harms ex Turn- } \\
\text { er }\end{array}$ & $2 n=9_{\mathrm{II}}$ & $\begin{array}{l}\text { MEXICO: Durango: } 5 \mathrm{mi} \mathrm{N} \text { of Durango, Pinkava } \\
\text { et al. } 13432 \text { pop. (ASU) }\end{array}$ \\
\hline $\begin{array}{l}\text { ** Heterotheca mucronata Harms ex } \\
\text { Turner }\end{array}$ & $2 n=9_{\mathrm{II}}$ & $\begin{array}{l}\text { MEXICO: Zacatecas, road above Concepción del } \\
\text { Oro, Pinkava et al. } 13486 \text { pop. (ASU) }\end{array}$ \\
\hline \multirow[t]{2}{*}{$\begin{array}{l}\text { Heterotheca villosa (Pursh) Shinners } \\
\text { var. foliosa (Nutt.) Harms }\end{array}$} & $n=9$ & $\begin{array}{l}\text { UNITED STATES: Arizona: Coconino Co.: San } \\
\text { Francisco Peaks, Pinkava et al. } 13756 \text { pop. } \\
\text { (ASU) }\end{array}$ \\
\hline & $n=18$ & $\begin{array}{l}\text { UNITED STATES: Texas: Culberson Co.: Lobos, } \\
\text { Reeves \& Pinkava } 13170 \text { (ASU); Arizona: Ya- } \\
\text { vapai Co.: Rt 179, } 5.6 \mathrm{mi} \text { N of jct I-17, P13897 } \\
\text { (ASU, OBI) }\end{array}$ \\
\hline $\begin{array}{l}\text { Heterotheca villosa (Pursh) Shinners } \\
\text { var. pedunculata (Greene) Harms ex } \\
\text { Semple }\end{array}$ & $2 n=9_{\mathrm{II}}$ & $\begin{array}{l}\text { UNITED STATES: Arizona: Apache Co.: } 9.5 \mathrm{mi} \\
\text { SE of Eager, Pinkava et al. } 12689 \text { (ASU) }\end{array}$ \\
\hline $\begin{array}{l}\text { Heterotheca villosa (Pursh) Shinners } \\
\quad \text { var. villosa }\end{array}$ & $n=18$ & $\begin{array}{l}\text { UNITED STATES: Utah: Grand Co.: Arches Na- } \\
\text { tional Park, K10854 pop. (ASU) [some cells } \\
\text { with lagging chromosomes at anaphase I, some } \\
\text { bridges and micronuclei]; Wyoming: Albany } \\
\text { Co.: I-80 at Buford exit, K10911 (ASU) }\end{array}$ \\
\hline $\begin{array}{l}{ }^{*} \text { Heterotheca villosa (Pursh) Shinners } \times \\
\text { H. fulcrata (Greene) Shinners }\end{array}$ & $2 n=3 x=9_{\mathrm{III}}$ & $\begin{array}{l}\text { UNITED STATES: New Mexico: Catron Co.: Rt } \\
\text { 180, } 2 \text { mi S of San Francisco River near Luna, } \\
\text { Pinkava et al. } 12752 \text { pop. (ASU) }\end{array}$ \\
\hline $\begin{array}{l}\text { Heterotheca aff. viscida (A. Gray) } \\
\text { Harms }\end{array}$ & $2 n=9_{\mathrm{II}}$ & $\begin{array}{l}\text { UNITED STATES: Arizona: Maricopa Co.: Just } \\
\text { S of Canyon Lake on Apache Trail, } K 11336 A \\
\text { (ASU) }\end{array}$ \\
\hline $\begin{array}{l}\text { **Lessingia germanorum Cham. var. } \\
\text { glandulifera (A. Gray) J. T. Howell }\end{array}$ & $2 n=5_{\mathrm{II}}+1_{\mathrm{I}}$ & $\begin{array}{l}\text { UNITED STATES: California: Kern Co.: } 3.2 \mathrm{mi} \\
\text { SE of jet of Keck and Twisselman Rds, } \\
M G K 11841-1 \text { (OBI) }\end{array}$ \\
\hline
\end{tabular}


TABLE 1. Continued

\begin{tabular}{|c|c|c|}
\hline $\begin{array}{l}\text { Tribe } \\
\text { Taxon or hybrid combination }\end{array}$ & Chromosome count & Location and voucher \\
\hline $\begin{array}{l}\text { *Lessingia nemaclada Greene var. albi- } \\
\quad \text { flora (Eastw.) J. T. Howell }\end{array}$ & $2 n=5_{\mathrm{II}}$ & $\begin{array}{l}\text { UNITED STATES: California: Kern Co.: Blue- } \\
\text { stone Ridge below Orchard Peak, } M G K 11845 \\
\text { (OBI) }\end{array}$ \\
\hline $\begin{array}{l}\text { Machaeranthera canescens (Pursh) A. } \\
\text { Gray }\end{array}$ & $2 n=4_{11}$ & $\begin{array}{l}\text { UNITED STATES: Arizona: Coconino Co.: San } \\
\text { Francisco Peaks, } P 13711 \text { (ASU), } P 13712 \text { (ASU) }\end{array}$ \\
\hline $\begin{array}{l}\text { Machaeranthera brevilingulata } \\
\text { (Hemsl.) Turner }\end{array}$ & $2 n=9_{\mathrm{II}}$ & $\begin{array}{l}\text { UNITED STATES: New Mexico: Hidalgo Co.. } 10 \\
\text { mi S of Hachita, KLL13425A (OBI) }\end{array}$ \\
\hline $\begin{array}{l}\text { Machaeranthera gracilis (Nutt.) Shin- } \\
\text { ners }\end{array}$ & $2 n=2_{\mathrm{II}}+1_{1}$ & $\begin{array}{l}\text { UNITED STATES: Arizona: Pima Co.: Molino } \\
\text { Basin, Santa Catalina Mtns, K12561 pop. (OBI) }\end{array}$ \\
\hline $\begin{array}{l}\text { Machaeranthera tanacetifolia (H.B.K.) } \\
\text { Nees }\end{array}$ & $2 n=4_{I I}$ & $\begin{array}{l}\text { UNITED STATES: Arizona: Pima Co.: Santa } \\
\text { Catalina Mtns, } K 12551 A(\mathrm{OBI})\end{array}$ \\
\hline $\begin{array}{l}\text { Machaeranthera tephrodes (A. Gray) } \\
\text { Greene }\end{array}$ & $2 n=5_{11}$ & $\begin{array}{l}\text { UNITED STATES: Arizona: Pima Co.: Molino } \\
\text { Basin, Santa Catalina Mtns, K12555 (OBI) }\end{array}$ \\
\hline $\begin{array}{l}\text { Pityopsis graminifolia (Michx.) Nutt. } \\
\text { var. latifolia (Fern.) Semple \& Bow- } \\
\text { ers }\end{array}$ & $2 n=18_{11}$ & $\begin{array}{l}\text { UNITED STATES: North Carolina: Cumberland } \\
\text { Co.: Ft. Bragg Military Reservation, K11609 } \\
\text { (ASU) }\end{array}$ \\
\hline Solidago confinis A. Gray & $2 n=9_{\mathrm{II}}$ & $\begin{array}{l}\text { UNITED STATES: California: San Luis Obispo } \\
\text { Co.: Montaña de Oro State Park, K11875 pop. } \\
\text { (OBI) }\end{array}$ \\
\hline Solidago missouriensis Nutt. & $2 n=9_{\mathrm{II}}$ & $\begin{array}{l}\text { UNITED STATES: Nebraska: Dawes Co.: } 12 \mathrm{mi} \\
\text { S of Crawford, K12855 (OBI); Wyoming: Al- } \\
\text { bany Co.: I-80 at Buford exit, K10906 (ASU) }\end{array}$ \\
\hline Solidago multiradiata Ait. & $2 n=9_{\mathrm{II}}$ & $\begin{array}{l}\text { UNITED STATES: Arizona: Coconino Co.: San } \\
\text { Francisco Peaks, } K 11547 \text { (ASU) }\end{array}$ \\
\hline Solidago odora Ait. & $2 n=9_{\mathrm{II}}$ & $\begin{array}{l}\text { UNITED STATES: North Carolina: Cumberland } \\
\text { Co.: Fort Bragg, } K 11664 \text { (ASU) }\end{array}$ \\
\hline $\begin{array}{l}\text { *Solidago patula Muhl. ex Willd. var. } \\
\text { strictula } \text { T. \& G. }\end{array}$ & $2 n=9_{11}$ & $\begin{array}{l}\text { UNITED STATES: North Carolina: Cumberland } \\
\text { Co.: Fort Bragg, } K 11681 A \text { (ASU) }\end{array}$ \\
\hline Solidago sparsiflora A. Gray & $2 n=9_{\mathrm{II}}$ & $\begin{array}{l}\text { UNITED STATES: Arizona: Coconino Co.: San } \\
\text { Francisco Peaks, P13715 (ASU); Santa Cruz } \\
\text { Co.: Peña Blanca Lake, K11029 (ASU); Yava- } \\
\text { pai Co.: Peeple's Valley, K11457 (ASU) }\end{array}$ \\
\hline Solidago wrightii A. Gray & $2 n=9_{\mathrm{II}}$ & $\begin{array}{l}\text { UNITED STATES: Arizona: Maricopa Co.: US } \\
602.6 \mathrm{mi} \text { E of Queen Creek Tunnel, L18903 } \\
\text { (ASU); Pima Co.: Molino Basin, Santa Catalina } \\
\text { Mtns, } K 12560(\mathrm{OBI})\end{array}$ \\
\hline $\begin{array}{l}\text { Xanthocephalum gymnospermoides (A. } \\
\text { Gray) Benth. var. gymnospermoides }\end{array}$ & $2 n=66_{\mathrm{II}}$ & $\begin{array}{l}\text { UNITED STATES: Arizona: } 2 \mathrm{mi} \mathrm{N} \text { of Elgin, } \\
K L L 13499 A(\mathrm{OBI})\end{array}$ \\
\hline \multicolumn{3}{|l|}{ CARDUEAE } \\
\hline $\begin{array}{l}{ }^{*} \text { Cirsium occidentale (Nutt.) Jeps. var. } \\
\text { compactum Hoover }\end{array}$ & $2 n=15_{\mathrm{II}}$ & $\begin{array}{l}\text { UNITED STATES: California: San Luis Obispo } \\
\text { Co.: N of San Simeon on Hwy } 1, K 11942 \text { (OBI) }\end{array}$ \\
\hline $\begin{array}{l}\text { Cirsium occidentale (Nutt.) Jeps. var. } \\
\quad \text { occidentale }\end{array}$ & $2 n=15_{\mathrm{II}}$ & $\begin{array}{l}\text { UNITED STATES: California: Monterey Co.: } 2 \\
\text { mi N of Arroyo Seco Rd on Carmel Rd, } \\
\text { K11951 (OBI) }\end{array}$ \\
\hline \multicolumn{3}{|l|}{ EUPATORIEAE } \\
\hline $\begin{array}{l}\text { Ageratina adenophora (Spreng.) King \& } \\
\text { Robinson }\end{array}$ & $2 n=3 x=51_{I}$ & $\begin{array}{l}\text { UNITED STATES: California: Monterey Co.: } 37 \\
\text { mi N of San Luis Obispo Co. line on Rt 1, } \\
\text { K11948 (OBI) }\end{array}$ \\
\hline $\begin{array}{l}\text { Agertina herbacea (A. Gray) King \& } \\
\text { Robinson }\end{array}$ & $2 n=17_{\mathrm{II}}$ & $\begin{array}{l}\text { UNITED STATES: Arizona: Cochise Co.: Monte- } \\
\text { zuma Pass, Huachuca Mtns, K13525 pop. } \\
\text { (OBI); Coconino Co.: Schnebly Hill Rd. } \\
\text { L20245 (ASU) }\end{array}$ \\
\hline Ageratum conyzoides $\mathrm{L}$. & $2 n=20_{\mathrm{II}}$ & $\begin{array}{l}\text { MARTINIQUE: ca. } 6 \mathrm{~km} \mathrm{~W} \text { of Gros Morne, } \\
\text { K16537 pop. (OBI) }\end{array}$ \\
\hline Ageratum corymbosum Zuccag. & $2 n=10_{\mathrm{II}}$ & $\begin{array}{l}\text { MEXICO: Sinaloa: } 4 \mathrm{mi} \text { N of Badiraguato, } \\
\text { KL15042 (OBI); Michoacán: Rt } 37,3 \mathrm{mi} \mathrm{S} \text { of } \\
\text { jct with Rt } 15, K L 15175(\mathrm{OBI})\end{array}$ \\
\hline Brickellia betonicifolia A. Gray & $2 n=9_{\text {II }}$ & $\begin{array}{l}\text { UNITED STATES: Arizona: Pima Co.: Madera } \\
\text { Canyon, Santa Rita Mtns, K12573 pop. (OBI) }\end{array}$ \\
\hline $\begin{array}{l}\text { *Brickellia chenopodina (Greene ex } \\
\text { Woot. \& Standl.) B. L. Robins. }\end{array}$ & $2 n=9_{\mathrm{II}}$ & $\begin{array}{l}\text { UNITED STATES: New Mexico: Hidalgo Co.: } 6 \\
\text { mi W of Antelope Wells, } K L L 13436 \text { pop. (OBI) }\end{array}$ \\
\hline
\end{tabular}


TABle 1. Continued

\begin{tabular}{|c|c|c|}
\hline $\begin{array}{l}\text { Tribe } \\
\text { Taxon or hybrid combination }\end{array}$ & Chromosome count & Location and voucher \\
\hline Brickellia grandiflora (Hook.) Nutt. & $2 n=9_{\text {II }}$ & $\begin{array}{l}\text { UNITED STATES: Arizona: Coconino Co.: San } \\
\text { Francisco Peaks, } P 13710 \text { (ASU), } P 13753 \text { (ASU) }\end{array}$ \\
\hline $\begin{array}{l}\text { *Koanophyllon villosum (Sw.) King \& } \\
\text { Robinson }\end{array}$ & $2 n=10_{11}$ & $\begin{array}{l}\text { JAMAICA: Hanover Parish: } 7 \mathrm{mi} \mathrm{E} \text { of Lucea, } \\
\text { K16392 pop. (OBI) }\end{array}$ \\
\hline Liatris squarrosa (L.) Michx. & $n=10$ & $\begin{array}{l}\text { UNITED STATES: North Carolina: Cumberland } \\
\text { Co.: Fort Bragg, } K 11697 \text { (ASU) }\end{array}$ \\
\hline Mikania micrantha Kunth & $2 n=19_{\mathrm{II}}$ & $\begin{array}{l}\text { JAMAICA: Westmoreland Parish: Negril, } \\
K 16403 A \text { (OBI) }\end{array}$ \\
\hline Mikania scandens (L.) Willd. & $2 n=18_{\mathrm{II}}$ & $\begin{array}{l}\text { UNITED STATES: North Carolina: Cumberland } \\
\text { Co.: Fort Bragg, } K 11635 \text { (ASU) }\end{array}$ \\
\hline Piqueria trinervia Cav. & $2 n=12_{\mathrm{II}}$ & $\begin{array}{l}\text { MEXICO: Michoacán: Rt } 37,3 \mathrm{mi} \text { S of jct with } \\
\text { Rt } 15, K L 15173 A(\mathrm{OBI}) \text {; México: } 4.5 \mathrm{mi} \text { W of } \\
\text { Santa María del Monte, } K L 15425 \text { (OBI) }\end{array}$ \\
\hline \multicolumn{3}{|l|}{ HELIANTHEAE } \\
\hline $\begin{array}{l}\text { Acanthospermum australe (Loefling) } \\
\text { Kuntze }\end{array}$ & $2 n=11_{\mathrm{II}}$ & $\begin{array}{l}\text { UNITED STATES: North Carolina: Cumberland } \\
\text { Co.: Fort Bragg, K11599 (ASU) }\end{array}$ \\
\hline Achyropappus anthemoides H.B.K. & $n=10$ & $\begin{array}{l}\text { MEXICO: México: } 4.5 \mathrm{mi} \mathrm{W} \text { of Santa María del } \\
\text { Monte on Rt } 1, K L 15420 \text { pop. (OBI) }\end{array}$ \\
\hline Acmella brachyglossa Cass. & $2 n=39_{1 I}$ & $\begin{array}{l}\text { MARTINIQUE: ca. } 6 \mathrm{~km} \text { W of Gros Morne, } \\
\text { K16535 pop. (OBI) }\end{array}$ \\
\hline $\begin{array}{l}\text { Acmella oppositifolia (Lam.) R. K. Jan- } \\
\text { sen var. oppositifolia }\end{array}$ & $2 n=26_{\text {II }}$ & $\begin{array}{l}\text { MEXICO: México: } 4.5 \mathrm{mi} \mathrm{W} \text { of Santa Maria del } \\
\text { Monte on Rt 1, KL15409 pop. (OBI); Michoa- } \\
\text { cán: } 1.1 \mathrm{mi} \mathrm{W} \text { Villamar, KL15152(OBI) }\end{array}$ \\
\hline Ambrosia confertiflora DC. & $2 n=54_{1 I}$ & $\begin{array}{l}\text { UNITED STATES: Arizona: Pima Co.: } 3 \mathrm{mi} \mathrm{W} \\
\text { of Tracy, K11807 (ASU) }\end{array}$ \\
\hline Bahia dissecta (A. Gray) Britton & $2 n=3 x=36$ & $\begin{array}{l}\text { UNITED STATES: Arizona: Coconino Co.: San } \\
\text { Francisco Peaks, P13691 (ASU). Meiosis very } \\
\text { irregular; multivalents present; pollen diads } \\
\text { rather than tetrads formed; many anthers with- } \\
\text { out sporogenous tissue or with only small bits } \\
\text { of sporogenous tissue }\end{array}$ \\
\hline \multirow[t]{2}{*}{ Baileya multiradiata $\mathrm{H} . \& \mathrm{G}}$. & $n=16$ & $\begin{array}{l}\text { UNITED STATES: Arizona: Cochise Co.: } 21.9 \\
\text { mi N of Pomerene, P10969 (ASU); Maricopa } \\
\text { Co.: Crater Mtns, P10025 (ASU); } 4 \text { mi S of Salt } \\
\text { River on Jackrabbit-Rainbow Rd, Sundell } 269- \\
\text { 1,-2,-4 (ASU); Pima Co.: Rt 386, } 0.5 \mathrm{mi} \mathrm{S} \text { of } \\
\text { jct Rt } 86, P 10726 \text { (ASU); Pinal Co.: } 8.1 \mathrm{mi} \mathrm{S} \text { of } \\
\text { Oracle, P11014 (ASU); Yuma Co.: I-8 at Spot } \\
\text { Rd exit, P10112, 10113 (ASU); } 4.1 \mathrm{mi} \mathrm{N} \text { of Al- } \\
\text { amo Crossing, P10295 (ASU); } 13 \mathrm{mi} \mathrm{N} \text { of Wen- } \\
\text { den, P10353 (ASU) }\end{array}$ \\
\hline & $2 n=16_{\mathrm{II}}+2_{\mathrm{II}} \mathrm{S}$ & $\begin{array}{l}\text { UNITED STATES: Árizona: Maricopa Co.: } 4 \mathrm{mi} \\
\text { S of Salt River on Jackrabbit-Rainbow Rd., } \\
\text { Sundell } 269-5 \text { (ASU) }\end{array}$ \\
\hline Baileya pauciradiata H. \& G. & $2 n=16_{\mathrm{II}}$ & $\begin{array}{l}\text { UNITED STATES: Arizona: Yuma Co.: Arizona } \\
\text { Western College, } P 10103-1,-2 \text { (ASU); Califor- } \\
\text { nia: Riverside Co.: W of Blythe, K11933 A, B } \\
\text { (OBI) }\end{array}$ \\
\hline Baileya pleniradiata H. \& G. & $n=16$ & $\begin{array}{l}\text { MEXICO: Baja California: Rosario, P11173 } \\
\text { (ASU); UNITED STATES: Arizona: Maricopa } \\
\text { Co.: White Tank Mtns, L17634 (ASU); Sierra } \\
\text { Estrella Mtns, P10115A, B (ASU) }\end{array}$ \\
\hline Bidens aequisquama (Fern.) Sherff & $2 n=36_{\text {II }}$ & $\begin{array}{l}\text { MEXICO: Michoacán: Rt } 37,3 \text { mi S of jet with } \\
\text { Rt } 15, K L 15176(\mathrm{OBI})\end{array}$ \\
\hline $\begin{array}{l}\text { Bidens alba (L.) DC. var. radiata (Sch. } \\
\text { Bip.) Ballard }\end{array}$ & $n=24$ & $\begin{array}{l}\text { UNITED STATES: Florida: along US } 4118 \mathrm{mi} \\
\text { W of jct with Florida Turnpike, K16478A (OBI) }\end{array}$ \\
\hline${ }^{* *}$ Bidens bipinnata $\mathrm{L}$ & $n=11$ & $\begin{array}{l}\text { MEXICO: Morelos: } 5.8 \mathrm{mi} \text { N of Tlaltizapán, } \\
\text { KL15459 (OBI) }\end{array}$ \\
\hline Bidens cynapifolia H.B.K. & $2 n=12_{\mathrm{II}}$ & $\begin{array}{l}\text { JAMAICA: St. Andrew's Parish: Beverley Hills } \\
\text { above Kingston, KB16413(OBI) }\end{array}$ \\
\hline $\begin{array}{l}\text { Bidens odorata Cav. var. calcicola } \\
\text { (Greenm.) Ballard }\end{array}$ & $2 n=12_{\mathrm{II}}$ & $\begin{array}{l}\text { MEXICO: Coahuila: rd past San Antonio, ca. } 30 \\
\text { mi E of jet Rt } 57, P 13752 \text { (ASU) }\end{array}$ \\
\hline $\begin{array}{l}\text { Bidens odorata Cav. aff. var. chilpan- } \\
\text { cingensis Ballard }\end{array}$ & $2 n=12_{\mathrm{II}}$ & $\begin{array}{l}\text { MEXICO: Guerrero, } 8.3 \mathrm{mi} \mathrm{N} \text { of Taxco, } \\
\text { KL } 15348 \text { (OBI) }\end{array}$ \\
\hline
\end{tabular}


TABle 1. Continued

\begin{tabular}{|c|c|c|}
\hline $\begin{array}{l}\text { Tribe } \\
\text { Taxon or hybrid combination }\end{array}$ & Chromosome count & Location and voucher \\
\hline $\begin{array}{l}\text { Bidens odorata Cav. var. oaxacensis } \\
\text { Ballard }\end{array}$ & $2 n=12_{\mathrm{II}}$ & $\begin{array}{l}\text { MEXICO: Oaxaca: } 13.1 \mathrm{mi} \text { S of Cuicatlán, } \\
\text { KLL15578A (OBI) }\end{array}$ \\
\hline Bidens pilosa L. var. pilosa & $2 n=36_{\mathrm{II}}$ & $\begin{array}{l}\text { MEXICO: Michoacán: Jiquilpan, } K L 15188(\mathrm{OBI}) \text {; } \\
\text { UNITED STATES: California: San Luis Obispo } \\
\text { Co.: California Polytechnic State University } \\
\text { campus, } K 11870(\mathrm{OBI})\end{array}$ \\
\hline **Bidens serrulata (Poir.) Desf. & $2 n=10_{\mathrm{II}}$ & $\begin{array}{l}\text { MEXICO: Oaxaca: } 8 \mathrm{mi} \mathrm{NW} \text { of Tamazulapan, } \\
\text { KL15501 (OBI) }\end{array}$ \\
\hline Bidens triplinervia H.B.K. & $2 n=24_{I I}$ & $\begin{array}{l}\text { MEXICO: México-Michoacán border on Rt } 15 \text {, } \\
\text { KL15600 (OBI) }\end{array}$ \\
\hline Calea ternifolia Kunth. var. ternifolia & $n=18$ & $\begin{array}{l}\text { MEXICO: Guerrero: } 2.5 \mathrm{mi} \text { S of Xolapa, } \\
\text { KL } 15313(\mathrm{OBI})\end{array}$ \\
\hline Chaenactis carphoclinia A. Gray & $2 n=8_{11}$ & $\begin{array}{l}\text { UNITED STATES: Arizona: La Paz Co.: I-10 at } \\
\text { Tom Wells Rd, K11937 pop. (OBI) }\end{array}$ \\
\hline $\begin{array}{l}\text { Chaenactis glabriuscula DC. var. lano- } \\
\text { sa (DC.) Hall }\end{array}$ & $2 n=6_{\mathrm{II}}$ & $\begin{array}{l}\text { UNITED STATES: California: San Luis Obispo } \\
\text { Co.: S of Shandon, } K 11953 A(\mathrm{OBI})\end{array}$ \\
\hline \multirow[t]{2}{*}{ Chaenactis stevioides H. \& A. } & $n=5$ & $\begin{array}{l}\text { UNITED STATES: Arizona: Maricopa Co.: } \\
\text { White Tank Mtns, K11203 (ASU) }\end{array}$ \\
\hline & $2 n=5_{\mathrm{II}}$ & $\begin{array}{l}\text { Yavapai Co.: jct Castle Creek Rd and rd to I-17, } \\
\text { L19745 (ASU); Lake Pleasant Regional Park, } \\
\text { L19715 (ASU) }\end{array}$ \\
\hline \multirow[t]{2}{*}{ Chaenactis xantiana A. Gray } & $2 n=5_{\mathrm{II}}$ & $\begin{array}{l}\text { UNITED STATES: California: Los Angeles Co.: } 5 \\
\text { mi W of San Bernardino Co. line on Rt } 138 \text {, } \\
\text { K11889 pop. (OBI) }\end{array}$ \\
\hline & $2 n=6_{\mathrm{II}}$ & $\begin{array}{l}\text { San Luis Obispo Co.: S of Shandon, } K 11954 A \\
\quad(\mathrm{OBI})\end{array}$ \\
\hline $\begin{array}{l}\text { Chrysanthellum indicum DC. var. } \\
\text { mexicanum (Greenm.) Turner }\end{array}$ & $2 n=8_{\text {II }}$ & $\begin{array}{l}\text { MEXICO: Nayarit: } 9 \mathrm{mi} \mathrm{E} \text { of turnoff to Compos- } \\
\text { tela on Hwy } 200, K L 15082 \text { pop. (OBI) }\end{array}$ \\
\hline $\begin{array}{l}\text { *Chrysanthellum michoacanum B. L. } \\
\text { Turner }\end{array}$ & $2 n=9_{\mathrm{II}}$ & $\begin{array}{l}\text { MEXICO: Michoacán: } 4.5 \text { mi S of Ápatzingán, } \\
\text { KL15237 (OBI) }\end{array}$ \\
\hline $\begin{array}{l}\text { Chrysanthellum involutum P. G. Wil- } \\
\text { son }\end{array}$ & $2 n=8_{\mathrm{II}}$ & $\begin{array}{l}\text { MEXICO: Guerrero: } 5.6 \mathrm{mi} \mathrm{E} \text { of turnoff to Arce- } \\
\text { lia on Rt } 51, K L 15368(\mathrm{OBI})\end{array}$ \\
\hline Coreopsis bigelovii (A. Gray) Hall & $2 n=12_{\mathrm{II}}$ & $\begin{array}{l}\text { UNITED STATES: California: San Luis Obispo } \\
\text { Co.: S of Shandon, } K 11952 A(\mathrm{OBI})\end{array}$ \\
\hline $\begin{array}{l}\text { Coreopsis californica (Nutt.) H. K. } \\
\text { Sharsm. var. californica }\end{array}$ & $n=12$ & $\begin{array}{l}\text { UNITED STATES: California: Los Angeles Co.: } 5 \\
\text { mi W of San Bernardino Co. line on Rt } 138 \text {, } \\
\text { K11891 pop. (OBI) }\end{array}$ \\
\hline **Cosmos caudatus H.B.K. & $2 n=12_{\mathrm{II}}$ & $\begin{array}{l}\text { MEXICO: Michoacán: } 7.2 \mathrm{mi} \text { SE of Huetamo, } \\
\text { KL15396 (OBI) }\end{array}$ \\
\hline Desmanthodium fruticosum Greenm. & $2 n=18_{\mathrm{II}}$ & $\begin{array}{l}\text { MEXICO: Guerrero: Rt } 95 \text { to Cuernavaca, } 7 \mathrm{mi} \mathrm{S} \\
\text { of jet with Rt } 55 \text { to Toluca, KL15356 (OBI) }\end{array}$ \\
\hline Dugaldia hoopesii (A. Gray) Rydb. & $2 n=15_{\mathrm{II}}$ & $\begin{array}{l}\text { UNITED STATES: Arizona: Coconino Co.: San } \\
\text { Francisco Peaks, K11528A (ASU), K11571 } \\
\text { (ASU) }\end{array}$ \\
\hline \multirow[t]{2}{*}{ Eriophyllum lanosum A. Gray } & $n=4$ & $\begin{array}{l}\text { UNITED STATES: Arizona: Maricopa Co.: Usery } \\
\text { Mtns, } L 17552 M \text { (ASU) }\end{array}$ \\
\hline & $2 n=4_{I I}$ & $\begin{array}{l}\text { Yavapai Co.: jct Castle Creek Rd and rd to I-17, } \\
\text { L19744 (ASU) }\end{array}$ \\
\hline *Flaveria angustifolia (Cav.) Pers. & $2 n=18_{\mathrm{II}}$ & $\begin{array}{l}\text { MEXICO: Puebla: } 5.5 \mathrm{mi} \mathrm{NW} \text { of Puebla-Oaxaca } \\
\text { border on Rt } 190, K L 15487 \text { (OBI) }\end{array}$ \\
\hline Flaveria linearis Lag. & $2 n=18_{\text {II }}$ & $\begin{array}{l}\text { UNITED STATES: Florida: Palm Beach Co.: } \\
\text { along Florida Turnpike at Lakeworth Plaza, } \\
\text { K16494 pop. (OBI) }\end{array}$ \\
\hline \multirow[t]{2}{*}{ Florestina pedata (Cav.) Cass. } & $2 n=10_{\mathrm{II}}$ & $\begin{array}{l}\text { MEXICO: Puebla: } 4.2 \mathrm{mi} \text { SE of Izúcar de Mata- } \\
\text { moros, KL15469 (OBI); Guerrero: } 7.9 \mathrm{mi} \mathrm{N} \text { of } \\
\text { Chilpancingo, KL15320 (OBI); Guerrero: } 7.1 \\
\text { mi E of Teloloapan, KL15366A (OBI) }\end{array}$ \\
\hline & $* * 2 n=11_{\mathrm{II}}$ & $\begin{array}{l}\text { MEXICO: Michoacán: } 4.2 \text { mi S of Nueva Italia, } \\
\text { KL15214 pop. (OBI) }\end{array}$ \\
\hline $\begin{array}{l}\text { Galinsoga parviflora Cav. var. parvi- } \\
\quad \text { flora }\end{array}$ & $2 n=8_{\mathrm{II}}$ & $\begin{array}{l}\text { MEXICO: México: } 4.5 \mathrm{mi} \text { W of Santa Maria del } \\
\text { Monte on Rt } 1, K L 15410 A(\mathrm{OBI})\end{array}$ \\
\hline $\begin{array}{l}\text { Galinsoga parviflora Cav. var. semical- } \\
\text { va A. Gray }\end{array}$ & $2 n=16_{\mathrm{II}}$ & $\begin{array}{l}\text { UNITED STATES: Arizona: Santa Cruz Co.: } \\
\text { Santa Rita Mtns, } 13.3 \text { mi E of Amado on road } \\
\text { to Mt. Hopkins, Keil \& Pinkava } 18504 \text { (OBI) }\end{array}$ \\
\hline
\end{tabular}


TABle 1. Continued

\begin{tabular}{|c|c|c|}
\hline $\begin{array}{l}\text { Tribe } \\
\text { Taxon or hybrid combination }\end{array}$ & Chromosome count & Location and voucher \\
\hline Helenium puberulum DC. & $2 n=29_{\mathrm{II}}$ & $\begin{array}{l}\text { UNITED STATES: California: San Luis Obispo } \\
\text { Co.: } 2 \text { mi E of Orcutt Rd on Moretti Canyon } \\
\text { Rd, K11811 (OBI) }\end{array}$ \\
\hline Helianthus annuus $\mathbf{L}$. & $n=17$ & $\begin{array}{l}\text { UNITED STATES: Arizona: Gila Co.: } 6.1 \mathrm{mi} \text { SW } \\
\text { of Seneca, L18927 (ASU); Wyoming: Bighorn } \\
\text { Co.: Yellowtail Lake, R. K. Brown 498-1 (ASU) }\end{array}$ \\
\hline Helianthus ciliaris DC. & $n=34$ & $\begin{array}{l}\text { UNITED STATES: Texas: Culberson Co.: Lobo, } \\
\text { P13167 (ASU) }\end{array}$ \\
\hline Helianthus debilis Nutt. & $2 n=17_{I I}$ & $\begin{array}{l}\text { UNITED STATES: Florida: Martin Co.: ca. } 1 \mathrm{mi} \\
\text { S of Jonathan Dickinson State Park along US 1, } \\
\text { K16497 pop. (OBI) }\end{array}$ \\
\hline Heliomeris hispida (A. Gray) Blake & $2 n=8_{\mathrm{II}}$ & $\begin{array}{l}\text { MEXICO: Sonora: Sierra de los Ajos, WNW of } \\
\text { Esqueda, } K L L 13588 A(\mathrm{OBI})\end{array}$ \\
\hline $\begin{array}{l}\text { Heliomeris multiflora Nutt. var. neva- } \\
\text { densis (A. Nels.) Yates }\end{array}$ & $2 n=8_{\text {II }}$ & $\begin{array}{l}\text { UNITED STATES: Arizona: Coconino Co.: San } \\
\text { Francisco Peaks, } P 13706 \text { (ASU) }\end{array}$ \\
\hline Heliopsis annua Hemsl. & $2 n=14_{I I}$ & $\begin{array}{l}\text { MEXICO: Michoacán: } 1.1 \mathrm{mi} \mathrm{W} \text { of Villemar, } \\
\text { KL15147 (OBI) }\end{array}$ \\
\hline $\begin{array}{l}\text { Hemizonia luzulifolia DC. ssp. rudis } \\
\text { (Benth.) Keck }\end{array}$ & $2 n=14_{1 I}$ & $\begin{array}{l}\text { UNITED STATES: California: Kern Co.: Blue- } \\
\text { stone Ridge below Orchard Peak, MGK11844 } \\
\text { (OBI) }\end{array}$ \\
\hline $\begin{array}{l}\text { Hemizonia pungens (H. \& A.) T. \& G. } \\
\text { ssp. pungens }\end{array}$ & $2 n=9_{\mathrm{II}}$ & $\begin{array}{l}\text { UNITED STATES: California: Kern Co.: Blue- } \\
\text { stone Ridge below Orchard Peak, MGK11850 } \\
\text { (OBI) }\end{array}$ \\
\hline Holocarpha heermannii (Greene) Keck & $2 n=66_{\mathrm{II}}$ & $\begin{array}{l}\text { UNITED STATES: California: Kern Co.: Blue- } \\
\text { stone Ridge below Orchard Peak, MGK11848 } \\
\text { (OBI) }\end{array}$ \\
\hline${ }^{*}$ Hymenothrix palmeri A. Gray & $2 n=12_{\mathrm{II}}$ & $\begin{array}{l}\text { MEXICO: Chihuahua: } 2 \mathrm{mi} \text { E of Colonia Pache- } \\
\text { co, KLL13348B (ASU, OBI) }\end{array}$ \\
\hline $\begin{array}{l}\text { Hymenoxys richardsonii (Hook.) Cock- } \\
\text { erell }\end{array}$ & $2 n=15_{\mathrm{II}}$ & $\begin{array}{l}\text { UNITED STATES: Arizona: Coconino Co.: San } \\
\text { Francisco Peaks, } P 13702 \text { (ASU) }\end{array}$ \\
\hline Lagascea mollis Cav. & $n=17$ & $\begin{array}{l}\text { ANTIGUA AND BARBUDA: Antigua: St. } \\
\text { John's Parish: } 1 \mathrm{mi} \text { SE of St Johns, K16551 } \\
\text { pop. (OBI) }\end{array}$ \\
\hline Lagophylla ramosissima Nutt. & $2 n=711$ & $\begin{array}{l}\text { UNITED STATES: California: San Luis Obispo } \\
\text { Co.: } 2 \text { mi E of Orcutt Rd along Moretti Canyon } \\
\text { Rd, K11812 (ASU, OBI) }\end{array}$ \\
\hline $\begin{array}{l}\text { Layia glandulosa (Hook.) H. \& A. var. } \\
\text { lutea (Keck) Hoover }\end{array}$ & $2 n=8_{\text {II }}$ & $\begin{array}{l}\text { UNITED STATES: California: San Luis Obispo } \\
\text { Co.: S of Shandon, K11955(OBI) }\end{array}$ \\
\hline Layia platyglossa (F. \& M.) A. Gray & $2 n=6_{11}$ & $\begin{array}{l}\text { UNITED STATES: California: San Luis Obispo } \\
\text { Co.: S of Shandon, K11956 pop. (OBI) }\end{array}$ \\
\hline $\begin{array}{l}\text { Melampodium divaricatum (Rich. in } \\
\text { Pers.) }\end{array}$ & $n=12$ & $\begin{array}{l}\text { MEXICO: Michoacán: Rt } 37,3 \text { mi S of jct with } \\
\text { Rt } 15, K L 15182 \text { (OBI) }\end{array}$ \\
\hline Melampodium gracile Less. & $2 n=9_{I I}$ & $\begin{array}{l}\text { MEXICO: Morelos: } 5.8 \mathrm{mi} \mathrm{N} \text { of Tlaltizapán on rd } \\
\text { to Cuautla, } K L 15461-1 \text { pop. (OBI) }\end{array}$ \\
\hline Melampodium pilosum Stuessy & $2 n=9_{\mathrm{II}}+1_{\mathrm{III}}$ & $\begin{array}{l}\text { MEXICO: Guerrero: } 4.8 \mathrm{mi} \mathrm{SW} \text { of Coyuca de Ca- } \\
\text { talan, } K L 15403 \text { pop. (OBI) }\end{array}$ \\
\hline Melanthera aspera (Jacq.) Small & $2 n=15_{I I}$ & $\begin{array}{l}\text { DOMINICAN REPUBLIC: Distrito Nacional: } \\
\text { Santo, KPr16425 pop. (OBI) }\end{array}$ \\
\hline Milleria quinqueflora $\mathrm{L}$. & $2 n=15_{11}$ & $\begin{array}{l}\text { MEXICO: Michoacán: } 11.5 \mathrm{mi} \text { E of Jiquilpan, } \\
\text { KL15145A (OBI) }\end{array}$ \\
\hline Montanoa tomentosa Cerv. & $2 n=19_{\mathrm{II}}+1 \mathrm{~s}$ & $\begin{array}{l}\text { MEXICO: Oaxaca: } 7.3 \mathrm{mi} \text { NW of Totolapan, } \\
K L 15534 \text { (OBI) }\end{array}$ \\
\hline $\begin{array}{l}\text { Otopappus imbricatus (Sch. Bip.) S. F. } \\
\text { Blake }\end{array}$ & $2 n=16_{I I}$ & $\begin{array}{l}\text { MEXICO: Guerrero: } 8.3 \mathrm{mi} \mathrm{N} \text { of Taxco, } \\
\text { KL15345 pop. (OBI) }\end{array}$ \\
\hline $\begin{array}{l}\text { Palafoxia arida Turner \& Morris var. } \\
\quad \text { arida }\end{array}$ & $2 n=12_{\mathrm{II}}$ & $\begin{array}{l}\text { UNITED STATES: Arizona: La Paz Co.: I-10 at } \\
\text { Tom Wells Rd, K11941 pop. (OBI); California: } \\
\text { Riverside Co.: E of Indio, K1 } 1878 \text { (OBI); Riv- } \\
\text { erside Co.: } 37 \text { mi E of Chiriaco Summit, } \\
\text { K11927 pop. (OBI) }\end{array}$ \\
\hline $\begin{array}{l}\text { Palafoxia linearis (Cav.) Lag. var. lin- } \\
\text { earis }\end{array}$ & $2 n=12_{11}$ & $\begin{array}{l}\text { MEXICO: Sinaloa: } 9 \mathrm{mi} \text { NW of El Dorado, } \\
\text { KL15057 (OBI) }\end{array}$ \\
\hline Perymenium macrocephalum Greenm. & $2 n=15_{\mathrm{II}}$ & $\begin{array}{l}\text { MEXICO: Guerrero: } 13 \mathrm{mi} \mathrm{N} \text { of Zumpango del } \\
\text { Río, KL15329 (OBI) }\end{array}$ \\
\hline
\end{tabular}


TABle 1. Continued

\begin{tabular}{|c|c|c|}
\hline $\begin{array}{l}\text { Tribe } \\
\text { Taxon or hybrid combination }\end{array}$ & Chromosome count & Location and voucher \\
\hline $\begin{array}{l}\text { Perymenium mendezii DC. var. men- } \\
\text { dezii }\end{array}$ & $3 n=\mathrm{ca} .45$ & $\begin{array}{l}\text { MEXICO: Oaxaca: } 8 \mathrm{mi} \mathrm{NW} \text { of Tamazulapan, } \\
\text { KL15504 (OBI) }\end{array}$ \\
\hline $\begin{array}{l}\text { Psilostrophe sparsiflora (A. Gray) A. } \\
\text { Nels. }\end{array}$ & $n=16$ & $\begin{array}{l}\text { UNITED STATES: Utah: Wayne Co.: Capital } \\
\text { Reef National Park, K10835 (ASU) }\end{array}$ \\
\hline \multirow[t]{2}{*}{ Psilostrophe tagetina (Nutt.) Greene } & $n=16$ & $\begin{array}{l}\text { UNITED STATES: New Mexico: Torrance Co.: } 7 \\
\text { mi E of Willard, } K 10727 \text { (ASU); Texas: El Paso } \\
\text { Co.: I- } 10 \text { at Clint turnoff, } P 13000 \text { (ASU) }\end{array}$ \\
\hline & $2 n=16_{\mathrm{II}}+1 \mathrm{~s}$ & $\begin{array}{l}\text { UNITED STATES: Arizona: Cochise Co.: } 2.5 \mathrm{mi} \\
\text { W of Portal, } K 8508-1 \text { (ASU; counted by R. C. } \\
\text { Brown) }\end{array}$ \\
\hline $\begin{array}{l}\text { Schkuhria anthemoidea (DC.) Coult. } \\
\quad \text { var. anthemoidea }\end{array}$ & $2 n=10_{\mathrm{II}}$ & $\begin{array}{l}\text { MEXICO: Nayarit: } 7 \mathrm{mi} \mathrm{NE} \text { of turnoff to Com- } \\
\text { postelo on Hwy } 200, K L 15086 \text { (OBI); Oaxaca: } \\
8 \mathrm{mi} \text { NW of Tamazulapan, } K L 15505 \text { (OBI) }\end{array}$ \\
\hline $\begin{array}{l}\text { *Schkuhria anthemoidea (DC.) Coult. } \\
\text { forma flava (Rydb.) Heiser }\end{array}$ & $2 n=10_{\mathrm{II}}$ & $\begin{array}{l}\text { MEXICO: Oaxaca: } 8 \mathrm{mi} \text { NW of Tamazulapan, } \\
\text { KL15506 (OBI) }\end{array}$ \\
\hline $\begin{array}{l}\text { Schkuhria anthemoidea (DC.) Coult. } \\
\text { var. wislizenii (A. Gray) Heiser }\end{array}$ & $2 n=20_{\mathrm{II}}$ & $\begin{array}{l}\text { MEXICO: Michoacán: Rt } 37,3 \text { mi S of jet with } \\
\text { Rt } 15, K L 15183 \text { (OBI) }\end{array}$ \\
\hline $\begin{array}{l}\text { Sclerocarpus divaricatus (Benth.) } \\
\text { Benth. \& Hook. ex Hemsl. }\end{array}$ & $2 n=11_{I I}$ & $\begin{array}{l}\text { MEXICO: Colima: } 4 \mathrm{mi} \mathrm{NW} \text { of jct Hwys } 200 \text { and } \\
\text { 110,KL15135 (OBI); Guerrero: } 7.8 \mathrm{mi} \mathrm{NW} \text { of } \\
\text { Petatlán, KL15291 (OBI) }\end{array}$ \\
\hline $\begin{array}{l}\text { *Sclerocarpus papposus (Greenm.) Fed- } \\
\text { dema }\end{array}$ & $2 n=13_{\mathrm{II}}$ & $\begin{array}{l}\text { MEXICO: Morelos: } 5.8 \mathrm{mi} \text { N of Tlaltizapán, } \\
\text { KL15458 (OBI) }\end{array}$ \\
\hline Sclerocarpus uniserialis (Hook.) Benth. & $2 n=12_{\mathrm{II}}$ & $\begin{array}{l}\text { MEXICO: Michoacán: } 0.2 \mathrm{mi} \mathrm{W} \text { of Cuatros Cam- } \\
\text { inos, KL15234 (OBI); Puebla: } 10.1 \mathrm{mi} \text { W of } \\
\text { Puebla-Oaxaca border on Rt } 190, K L 15486 \\
\text { (OBI) }\end{array}$ \\
\hline $\begin{array}{l}\text { ** Silphium compositum Michx. } \times(?) S \text {. } \\
\text { asteriscus } \text { Ell. }\end{array}$ & $2 n=7_{\mathrm{II}}+1_{\mathrm{I}}$ & $\begin{array}{l}\text { UNITED STATES: North Carolina: Cumberland } \\
\text { Co.: Fort Bragg, K11632 (ASU). Pollen stain- } \\
\text { ability } 3.8 \% \text {; macropollen and micropollen } \\
\text { present }\end{array}$ \\
\hline Simsia lagasciformus DC. & $2 n=17_{\mathrm{II}}$ & $\begin{array}{l}\text { MEXICO: Guerrero: } 15 \mathrm{mi} \mathrm{N} \text { of Zumpango del } \\
\text { Río, } K L 15419 \text { (OBI) }\end{array}$ \\
\hline Spilanthes urens Jacq. & $n=16$ & $\begin{array}{l}\text { MEXICO: Jalisco: Puerto Vallarta, KL15103 } \\
\text { (OBI) JAMAICA: St. James Parish: Montego } \\
\text { Bay, K16388 pop. (OBI) }\end{array}$ \\
\hline *Stuessya michoacana Turner \& Davies & $2 n=17_{\mathrm{II}}$ & $\begin{array}{l}\text { MEXICO: Michoacán: } 6 \text { mi S of Gabriel Zamora, } \\
\text { KL15212 (OBI) }\end{array}$ \\
\hline Stuessya perennans Turner \& Davies & $2 n=17_{11}$ & $\begin{array}{l}\text { MEXICO: Guerrero: } 5.6 \mathrm{mi} \mathrm{W} \text { of turnoff to } \mathrm{La} \\
\text { Union on Rt } 200, K L 15286(\mathrm{OBI})\end{array}$ \\
\hline $\begin{array}{l}\text { Thelesperma megapotamicum (Spreng.) } \\
\text { Kuntze }\end{array}$ & $2 n=22_{11}$ & $\begin{array}{l}\text { UNITED STATES: Arizona: Pima Co.: Luckow } \\
633 \text { (OBI) }\end{array}$ \\
\hline Tithonia pedunculata Cronq. & $2 n=17_{11}$ & $\begin{array}{l}\text { MEXICO: Oaxaca: } 9.8 \mathrm{mi} \mathrm{NW} \text { of El Coyula on } \\
\text { Rt } 190, K L 15544 \text { (OBI) }\end{array}$ \\
\hline $\begin{array}{l}\text { Trichoptilium incisum (A. Gray) A. } \\
\text { Gray }\end{array}$ & $2 n=13_{11}$ & $\begin{array}{l}\text { UNITED STATES: California: Riverside Co.: } \\
\text { Cactus City rest area along I-10, K11929 pop. } \\
\text { (OBI) }\end{array}$ \\
\hline Tridax coronopifolia (H.B.K.) Hemsl. & $2 n=9_{1 I}$ & $\begin{array}{l}\text { MEXICO: Guerrero: } 7.8 \mathrm{mi} \text { NW of Petatlán, } \\
\text { KL15292 (OBI) }\end{array}$ \\
\hline Tridax platyphylla B. L. Robins. & $2 n=9_{\mathrm{II}}$ & $\begin{array}{l}\text { MEXICO: Michoacán: } 11.6 \mathrm{mi} \mathrm{N} \text { of Nuevo Cen- } \\
\text { tro, } K L 15258(\mathrm{OBI}) \text {; Guerrero: } 2.1 \mathrm{mi} \mathrm{N} \text { of Río } \\
\text { Mezcala on Rt } 95, K L 15335(\mathrm{OBI})\end{array}$ \\
\hline $\begin{array}{l}\text { Verbesina encelioides (Cav.) A. Gray } \\
\text { ssp. encelioides }\end{array}$ & $2 n=17_{I I}$ & $\begin{array}{l}\text { DOMINICAN REPUBLIC: Monte Christi Prov- } \\
\text { ince: S of Monte Christi along road to Dajabón, } \\
\text { KPr16432 pop. (OBI) }\end{array}$ \\
\hline Viguiera grammatoglossa DC. & $2 n=17_{11}$ & $\begin{array}{l}\text { MEXICO: Oaxaca: } 7.7 \mathrm{mi} \text { W of Tamazulapan, } \\
\text { KL } 15511 \text { (OBI) }\end{array}$ \\
\hline Viguiera microcephala Greenm. & $2 n=12_{I I}$ & $\begin{array}{l}\text { MEXICO: Oaxaca: } 12 \mathrm{mi} \text { SE of Totolapan, } \\
\text { KL15540 (OBI) }\end{array}$ \\
\hline${ }^{*}$ Wedelia calycina L. C. Rich. & $n=24$ & $\begin{array}{l}\text { ANTIGUA AND BARBUDA: Antigua: St. Paul's } \\
\text { Parish: Falmouth Harbour, K16549 pop. (OBI) }\end{array}$ \\
\hline${ }^{*}$ Wedelia aff. ehrenbergii Schlecht. & $2 n=24_{I I}$ & $\begin{array}{l}\text { DOMINICAN REPUBLIC: Dajabón Province: } \\
\text { Santiago de la Cruz, KPr16441 (OBI) }\end{array}$ \\
\hline *Wedelia reticulata DC. & $n=14$ & $\begin{array}{l}\text { DOMINICAN REPUBLIC: Puerto Plata Prov- } \\
\text { ince: S of Imbert, K16427 pop. (OBI) }\end{array}$ \\
\hline
\end{tabular}


TABLE 1. Continued

\begin{tabular}{|c|c|c|}
\hline $\begin{array}{l}\text { Tribe } \\
\text { Taxon or hybrid combination }\end{array}$ & Chromosome count & Location and voucher \\
\hline${ }^{*}$ Wedelia sp. nov. & $2 n=22_{\mathrm{II}}$ & $\begin{array}{l}\text { MEXICO: Michoacán: } 16.3 \mathrm{mi} \mathrm{S} \text { of Uruapan, } \\
\text { KL15201 (OBI) }\end{array}$ \\
\hline Zinnia angustifolia H.B.K. & $n=11$ & $\begin{array}{l}\text { MEXICO: Jalisco: } 8 \mathrm{mi} \mathrm{N} \text { of El Tuito, KL15110 } \\
\text { (OBI) }\end{array}$ \\
\hline Zinnia palmeri A. Gray in S. Wats. & $2 n=12_{\mathrm{II}}$ & $\begin{array}{l}\text { MEXICO: Jalisco: Puerto Vallarta, KL15102 } \\
\text { (OBI) }\end{array}$ \\
\hline **Zinnia tenella $\mathrm{B}$. L. Robins. & $2 n=10_{\mathrm{II}}$ & $\begin{array}{l}\text { MEXICO: Michoacán: } 7.4 \mathrm{mi} \mathrm{N} \text { of Nuevo Cen- } \\
\text { tro, KL15260 (OBI) }\end{array}$ \\
\hline \multicolumn{3}{|l|}{ INULEAE } \\
\hline *Gnaphalium californicum DC. & $2 n=14_{I I}$ & $\begin{array}{l}\text { UNITED STATES: California: San Luis Obispo } \\
\text { Co.: See Canyon, } K 11835 \text { (OBI) }\end{array}$ \\
\hline Gnaphalium canescens DC. & $2 n=14_{I I}$ & $\begin{array}{l}\text { UNITED STATES: Arizona: Pima Co.: Madera } \\
\text { Canyon, Santa Rita Mtns, K12577 pop. (OBI) }\end{array}$ \\
\hline Gnaphalium exilifolium A. Nels. & $2 n=7_{\mathrm{II}}$ & $\begin{array}{l}\text { UNITED STATES: Arizona: Coconino Co.: San } \\
\text { Francisco Peaks, } P 13697 \text { (ASU) }\end{array}$ \\
\hline Gnaphalium leucocephalum A. Gray & $2 n=14_{1 I}$ & $\begin{array}{l}\text { UNITED STATES: Arizona: Pima Co.: Santa } \\
\text { Catalina Mtns, } K 12562 \text { (OBI) }\end{array}$ \\
\hline Gnaphalium palustre Nutt. & $2 n=7_{\mathrm{II}}$ & $\begin{array}{l}\text { UNITED STATES: California: San Luis Obispo } \\
\text { Co.: Lopez Lake, } K 13661 A(\mathrm{OBI})\end{array}$ \\
\hline Pluchea odorata (L.) Cass. & $2 n=10_{\mathrm{II}}$ & $\begin{array}{l}\text { ANTIGUA AND BARBUDA: Antigua: St. } \\
\text { George's Parish: between Coolidge Airport and } \\
\text { St. George's Church K16557 pop. (OBI) }\end{array}$ \\
\hline Pluchea symphytifolia (Mill.) Gillis & $2 n=10_{\mathrm{II}}$ & $\begin{array}{l}\text { ANTIGUA AND BARBUDA: Antigua: St. } \\
\text { George's Parish: between Coolidge Airport and } \\
\text { St. George's Church K16556 pop. (OBI) }\end{array}$ \\
\hline \multicolumn{3}{|l|}{ LACTUCEAE } \\
\hline Agoseris aurantiaca (Hook.) Greene & $2 n=17_{\mathrm{II}}$ & $\begin{array}{l}\text { UNITED STATES: Arizona: Coconino Co.: San } \\
\text { Francisco Peaks, } P 13768 \text { pop. (ASU) }\end{array}$ \\
\hline Agoseris glauca (Pursh) D. Dietr. & $2 n=18_{\mathrm{II}}$ & $\begin{array}{l}\text { UNITED STATES: Arizona: Coconino Co.: San } \\
\text { Francisco Peaks, } K 11527 A \text { (ASU) }\end{array}$ \\
\hline Agoseris grandiflora (Nutt.) Greene & $2 n=9_{11}$ & $\begin{array}{l}\text { UNITED STATES: California: San Luis Obispo } \\
\text { Co.: See Canyon, } K 11834 \text { (OBI) }\end{array}$ \\
\hline Hieracium gronovii $\mathrm{L}$. & $2 n=9_{\mathrm{II}}$ & $\begin{array}{l}\text { UNITED STATES: North Carolina: Cumberland } \\
\text { Co.: Fort Bragg, K11640 (ASU), K11656 (ASU) }\end{array}$ \\
\hline${ }^{*}$ Hieracium pringlei A. Gray & $2 n=9_{\mathrm{II}}$ & $\begin{array}{l}\text { MEXICO: Michoacán: Rt } 37,10 \text { mi S of jct with } \\
\text { Rt } 15, K L 15190(\mathrm{OBI})\end{array}$ \\
\hline Hypochoeris radicata $\mathrm{L}$. & $2 n=4_{1 I}$ & $\begin{array}{l}\text { UNITED STATES: North Carolina: Cumberland } \\
\text { Co.: Fort Bragg, K11621 pop. (ASU) }\end{array}$ \\
\hline Lactuca saligna $\mathrm{L}$. & $2 n=9_{11}$ & $\begin{array}{l}\text { UNITED STATES: California: San Luis Obispo } \\
\text { Co.: } 2.3 \mathrm{mi} \mathrm{N} \text { of Righetti Rd on Orcutt Rd, } \\
\text { K11 } 1809(\mathrm{OBI})\end{array}$ \\
\hline Malacothrix incana (Nutt.) T. \& G. & $2 n=7_{\mathrm{II}}$ & $\begin{array}{l}\text { UNITED STATES: California: San Luis Obispo } \\
\text { Co.: Montaña de Oro State Park, MMK } 11860 \\
\text { (OBI) }\end{array}$ \\
\hline $\begin{array}{l}\text { Microseris heterocarpa (Nutt.) Cham- } \\
\text { bers }\end{array}$ & $2 n=18_{\mathrm{II}}$ & $\begin{array}{l}\text { UNITED STATES: California: Riverside Co.: Al- } \\
\text { berthill Jct along Hwy } 71, M K 12967 \text { pop. (OBI) }\end{array}$ \\
\hline Pinaropappus roseus (Less.) Less. & $2 n=16_{\mathrm{II}}+1_{\mathrm{IV}}$ & $\begin{array}{l}\text { MEXICO: Oaxaca: } 7.7 \mathrm{mi} \text { SE of Tamazulapan on } \\
\text { Rt } 190, K L 15510 \text { (OBI) }\end{array}$ \\
\hline $\begin{array}{l}\text { Pyrrhopappus carolinianus (Walt.) DC. } \\
\text { var. carolinianus }\end{array}$ & $2 n=6_{\mathrm{II}}$ & $\begin{array}{l}\text { UNITED STATES: North Carolina: Cumberland } \\
\text { Co.: Fort Bragg, } K 11637 \text { (ASU) }\end{array}$ \\
\hline Stephanomeria cichoriacea A. Gray & $2 n=8_{I I}$ & $\begin{array}{l}\text { UNITED STATES: California: San Luis Obispo } \\
\text { Co.: Prefumo Canyon, } K 11832(\mathrm{OBI})\end{array}$ \\
\hline Stephanomeria thurberi A. Gray & $2 n=32$ & $\begin{array}{l}\text { UNITED STATES: Arizona: Coconino Co.: San } \\
\text { Francisco Peaks, } P 13755 \text { (ASU). } 2 n=11_{\mathrm{II}}+ \\
1_{\mathrm{IV}}+1_{\mathrm{VI}} \text {; multivalents vary from cell to cell; } \\
\text { some cells with univalents }\end{array}$ \\
\hline Tragopodon dubius Scop. & $n=6$ & $\begin{array}{l}\text { UNITED STATES: Arizona: Apache Co.: Green's } \\
\text { Mountain Rd, } 4.8 \text { mi S of jct with Rt } 60 \text {, } \\
\text { P11513 (ASU); Coconino Co.: Flagstaff, Cedar } \\
\text { Dr. near municipal watertower, } R \text {. K. Brown } \\
215-2,-3 ; 229-1,-7 ; 416-4 ; 428-8 \text { (all ASU); }\end{array}$ \\
\hline
\end{tabular}


TABle 1. Continued

\begin{tabular}{lcc}
\hline \hline $\begin{array}{l}\text { Tribe } \\
\text { Taxon or hybrid combination }\end{array}$ & Chromosome count & Location and voucher \\
\hline
\end{tabular}

Tragopogon mirus Owenbey

$n=12$

Tragopogon porrifolius $\mathrm{L}$.

SENECIONEAE

Senecio blochmaniae Greene

Senecio douglasii DC. var. monoensis

(Greene) Jeps.

Senecio eremophilus Rich. var. macdougalii (Heller) Cronq.

Senecio lemmonii A. Gray

Senecio multicapitatus Greenm.

Senecio vulgaris $\mathrm{L}$.

\section{TAGETEAE}

Dyssodia papposa (Vent.) Hitch.

Dyssodia decipiens (Bartling) M. C. Johnston \& B. L. Turner

Dyssodia tagetiflora Lag.

*Pectis canescens H.B.K.

*Pectis carthusianorum Less.

Pectis cylindrica (Fern.) Rydb.

*Pectis decemcarinata McVaugh

$2 n=12_{\mathrm{II}}$
Flagstaff, Rt $180 \mathrm{NW}$ of Plaza Mall, $L 17780$ (ASU); Lindburg Spring Road, Rt 89A, L17735-6 (ASU); Lake Mary, L17765-1 (ASU); Gila Co.: Rt 60-77, ca. $26 \mathrm{mi} \mathrm{N}$ of Globe, $R$. $K$. Brown 610-2 (ASU); Kohl's Ranch, Rt 260, $R$. K. Brown 700-1, -4 (ASU); Greenlee Co.: $13 \mathrm{mi}$ S of Strayhorse campground, P11288 (ASU); Navajo Co.: Woodland Rd. and Rt 260, Lakeside, $R$. K. Brown 608 (ASU); Yavapai Co.: Prescott, R. K. Brown 234-1 (ASU); 2 mi E of Prescott Community College, Rt 69, R. K. Brown 699-1, -3 (ASU)

UNITED STATES: Arizona: Flagstaff, Rt 180 NW of Plaza Mall, $R$. K. Brown 425-2, -4, -31; 427-3 (all ASU); Flagstaff, Cedar Dr., near municipal watertower, $R$. K. Brown 214-1; 227-4, $-5,-6 ; 390-4-1,-6$ (yellow-flowered form); 391 1; 418-2 (aberrant capitulum; $86.4 \%$ pollen stainability) (all ASU)

UNITED STATES: Arizona: Coconino Co.: Flagstaff, near municipal watertower, $R$. K. Brown 216-1, -2; 230-1 (ASU)

UNITED STATES: California: San Luis Obispo Co.: Morro Bay, MMK11857 (OBI)

UNITED STATES: Arizona: Yavapai Co.: I-17, $0.5 \mathrm{mi}$ N of Sunset Point, L19930 (ASU)

UNITED STATES: Arizona: Coconino Co.: San Francisco Peaks, P13730 (ASU), P13778 (ASU)

UNITED STATES: Arizona: Maricopa Co.: White Tank Mtns, K11190 (ASU)

UNITED STATES: Arizona: Coconino Co.: San Francisco Peaks, P13714 (ASU)

UNITED STATES: Arizona: Maricopa Co.: Metrocenter, Phoenix, K11325 (ASU)

BOLIVIA: Chuquisaca: $12 \mathrm{~km}$ NE of Sucre, Canne 2767 (OBI); MEXICO: México: $4.5 \mathrm{mi}$ W of Santa María del Monte, KL15411 (OBI)

MEXICO: Oaxaca: $2.1 \mathrm{mi}$ SE of Mazatlán, KL15531 (OBI)

MEXICO: Guerrero: $8.3 \mathrm{mi} \mathrm{N}$ of Taxco, KL15344 (OBI)

MEXICO: Guerrero: 4.3 mi SE of jct with Rt 200 on rd to Barra Vieja, KL15302A, pop. (OBI)

DOMINICAN REPUBLIC: Distrito Nacional: Jardín Botánico Nacional, KPr16421 A.D (OBI)

MEXICO: Sinaloa: $0.3 \mathrm{mi} \mathrm{W}$ of Las Cascabeles, $K L 15052$ pop. (OBI); Sonora: $15.4 \mathrm{mi} \mathrm{N}$ of turnoff to Bahia Kino on Hwy 15, KL15001 pop. (OBI)

MEXICO: Michoacán: $4.2 \mathrm{~m}$ N of Nueva Italia, $K L 15216 A, B, C$ (OBI); $5.5 \mathrm{mi} \mathrm{N}$ of Nueva Italia, KL15229 pop. (OBI); Cuatros Caminos, $K L 15232$ pop. (OBI); $2.3 \mathrm{mi} \mathrm{S}$ of Apatzingán, KL15236A (OBI); $7.2 \mathrm{mi} \mathrm{S}$ of Apatzingán $K L 15238$ pop. (OBI); $1.5 \mathrm{mi} \mathrm{N}$ of Santa Ana Amatlán, $K L 15240$ pop. (OBI); $3 \mathrm{mi} \mathrm{W}$ of Apatzingán, $K L 15241 C, D, F, G(\mathrm{OBI}) ; 8.9 \mathrm{mi} \mathrm{N}$ of Nuevo Centro, KL15259A (OBI)

Pectis depressa Fern.

$2 n=12_{\text {II }}$

MEXICO: Guerrero: $7.8 \mathrm{mi} \mathrm{NW}$ of Petatlán, 
TABle 1. Continued

\begin{tabular}{lcc}
\hline \hline $\begin{array}{l}\text { Tribe } \\
\text { Taxon or hybrid combination }\end{array}$ & Chromosome count & Location and voucher \\
\hline
\end{tabular}

*Pectis diffusa Hook. \& Arn.

* Petis elongata H.B.K. var. floribunda (A. Rich.) Keil

*Pectis elongata H.B.K. var. floribunda (A. Rich.) Keil $\times P$. humifusa Sw.

*Pectis elongata H.B.K. var. floribunda (A. Rich.) Keil $\times P$. linearis Llave

*Pectis exserta McVaugh

Pectis filipes H. \& G. ex A. Gray var. subnuda Fern.

Pectis $\times$ floridana Keil ( $P$. glaucescens (Cass.) Keil $\times$ P. prostrata Cav.)

Pectis glaucescens (Cass.) Keil

*Pectis humifusa $\mathrm{Sw}$.
$2 n=4 x=48$

$2 n=24$

$2 n=12_{\text {II }}$

$2 n=12_{\text {II }}$

$2 n=3 x=36$

$2 n=24_{\text {II }}$

$2 n=36_{\text {II }}$
$K L 15290 A, C$ (OBI); $4.1 \mathrm{mi}$ NW of Petatlán, $K L 15293 A, B(\mathrm{OBI}) ; 8 \mathrm{mi} \mathrm{S}$ of Acapulco on rd to Barra Vieja, KL15300 pop. (OBI); El Bejuco, $K L 15307 A, B$ (OBI)

MEXICO: Jalisco: N of Guadalajara on Rt 54, $K L 15631 A, B, D$ (OBI)

DOMINICAN REPUBLIC: El Siebo Prov.: $5 \mathrm{~km}$ $\mathrm{S}$ of Hato Mayor, $K P r 16442 A, C$ (OBI); JAMAICA: Hanover Parish: $7 \mathrm{mi}$ E of Lucea, K16390 pop. (OBI); Hanover Parish: $2 \mathrm{mi} \mathrm{E}$ of Lucea, K16405A (OBI); MARTINIQUE: $2 \mathrm{mi}$ E of Trois Islets, K16522 pop. (OBI); $22 \mathrm{~km} \mathrm{NE}$ of Marin, K16525 pop. (OBI); at turnoff to St. Anne along rd from Marin to Salines, K16526 pop. (OBI); PUERTO RICO: $4.5 \mathrm{mi} \mathrm{W}$ of Coamo, K16518A,B (OBI); $1.5 \mathrm{mi} \mathrm{NE}$ of Rincón, $K 16505 A$ (OBI); $3 \mathrm{mi}$ SSE of Rincón, $K 16508 A$ (OBI)

MARTINIQUE: $22 \mathrm{~km}$ NE of Marin, K16524C (OBI). Meiosis is very irregular (e.g., $1_{\mathrm{IV}}+5_{\mathrm{II}}+$ 34)

PUERTO RICO: $1.5 \mathrm{mi} \mathrm{NE}$ of Rincón, K16507 (OBI). Some cells have apparently normal meiosis; others at metaphase I have univalents or early disjunction; some anaphase I cells have a bridge + a fragment

MEXICO: Jalisco: $4 \mathrm{mi} \mathrm{N}$ of Río Tomatlán on Hwy $200, K L 15117 B, C ; 1 \mathrm{mi}$ S of Río San Nicolas on Hwy 200, KL15119A,B,D,E (OBI); $3 \mathrm{mi}$ from Playa la Fortuna, $K L 15120 A, B, C(\mathrm{OBI})$; Barra de Navidad, $K L 15126 A, B, C$ (OBI)

UNITED STATES: Arizona: Pima Co.: Santa Catalina Mtns, K12552 pop. (OBI); Santa Cruz Co.: $5 \mathrm{mi}$ from jet with Lochiel-Nogales Rd on rd from Patagonia, K12597 pop. (OBI)

UNITED STATES: Florida: Dade Co.: US 41, W of Florida Turnpike, K16476B (OBI); Collier Co.: $6 \mathrm{mi}$ SE of Royal Palm Hammock, $K 16488 A, C, D(\mathrm{OBI})$. Meiosis is very irregular with a variable number of I's, II's, III's, and IV's

DOMINICAN REPUBLIC: Distrito Nacional: Santo Domingo, Jardín Botánico Nacional, KPr16420 pop. (OBI), 16422 pop. (OBI); JAMAICA: St. Elizabeth Parish: 1 mi N of Mountainside, K16426 pop. (OBI); UNITED STATES: Florida: Collier Co.: $6 \mathrm{mi}$ SE of Royal Palm Hammock, K16487 A,B (OBI) Dade Co.: US $4118 \mathrm{mi} \mathrm{W}$ of Florida Turnpike, $K 16475 A, B, C$ (OBI); US $414 \mathrm{mi} \mathrm{W}$ of Shark Valley K16481 pop. (OBI); US $413 \mathrm{mi}$ E of Monroe Station, K16482 pop. (OBI); US 410.5 mi W of Carnestown, K16484 pop. (OBI); Martin Co., $5.5 \mathrm{mi} \mathrm{N}$ of Hobe Sound, K16501 pop. (OBI); Palm Beach Co.: Florida Turnpike at Lakeworth Service Plaza, K16501 pop. (OBI)

ANTIGUA AND BARBUDA: Antigua: St. Paul's Parish: Falmouth Harbour, K16548C,D,E (OBI); St. George's Parish: Windthorpe's Bay, K16552 pop. (OBI); MARTINIQUE: Salines, K16527 pop. (OBI); $4 \mathrm{~km} \mathrm{~N}$ of Vauclin, K16530 pop. (OBI); PUERTO RICO: Isla de Cabra, K16502A,C (OBI) 
TABle 1. Continued

\begin{tabular}{lcc}
\hline Tribe & Chromosome count & Location and voucher \\
Taxon or hybrid combination
\end{tabular}

Pectis imberbis A. Gray $2 n=12_{\mathrm{II}}$

**Pectis latisquama Sch. Bip. ex Greenm. $2 n=72$

Pectis leavenworthii Standl.

$2 n=12_{\mathrm{II}}$

Pectis linearifolia Urban

*Pectis linearis Llave

*Pectis linifolia L. var. hirtella S. F. $\quad 2 n=12_{\mathrm{II}}$ Blake

Pectis linifolia L. var. linifolia

$2 n=12_{\mathrm{II}}$

Pectis multiflosculosa (DC.) Sch. Bip. $\quad 2 n=36_{\mathrm{II}}$

Pectis papposa H. \& G. ex A. Gray

var. papposa

Pectis prostrata Cav.

Pectis purpurea Brandegee var. sonorae Keil

*Pectis schaffneri Sch. Bip. ex Fern. $\quad 2 n=12_{\mathrm{II}}$

Pectis saturejoides (Mill.) Sch. Bip. $2 n=12_{\mathrm{II}}$

*Pectis sessiliflora (Less.) Sch. Bip. $\quad 2 n=24_{\text {II }}$

*Pectis tenuicaulis Urban
UNITED STATES: Arizona: Cochise Co.: S end of Canelo Hills, KLL13533 (OBI)

MEXICO: Puebla: Tepeala, KL15593 pop. (OBI); $3.9 \mathrm{mi}$ SE of Amazoc, KL15599A,C,D (OBI). Meiosis is nearly normal with mostly bivalent pairing and a variable number of univalents

MEXICO: Michoacán: $5 \mathrm{mi} \mathrm{N}$ of Gabriel Zamora, KL15209 pop. (OBI); $2.6 \mathrm{mi} \mathrm{N}$ of Gabriel Zamora, KL15225 pop. (OBI); $2.3 \mathrm{mi} \mathrm{S}$ of Apatzingán, $K L 15236 B(\mathrm{OBI})$; Nueva Italia, KL15231 pop. (OBI)

UNITED STATES: Florida: Martin Co.: $5.5 \mathrm{mi} \mathrm{N}$ of Hobe Sound, $K 16500 A$, pop. (OBI)

MEXICO: Oaxaca: $14.7 \mathrm{mi} \mathrm{W}$ of Niltepec, $K L 15558$ pop. (OBI); PUERTO RICO: $1.5 \mathrm{mi}$ NE of Rincón, $K 16506 A, B(\mathrm{OBI})$

MEXICO: Michoacán: $7 \mathrm{mi}$ W of Cuatros Caminos, $K L 15217 A, B, C$ (OBI); $8 \mathrm{mi} \mathrm{S}$ Cuatros Caminos, $K L 15248$ pop. (OBI)

MEXICO: Oaxaca: $3 \mathrm{mi} \mathrm{NW}$ of Totolapan, KL15535 pop. (OBI); $15.1 \mathrm{mi}$ SE of Totolapan, $K L 15542$ pop. (OBI); PERU: Lambayeque: 28 $\mathrm{km} \mathrm{W}$ of Olmos, Dillon 1721 progeny (OBI); PUERTO RICO: $4.4 \mathrm{mi}$ SE of Boqueron, $K 16509 A, B, C, D(\mathrm{OBI}) ; 1 \mathrm{mi} \mathrm{W}$ of Ensenada, $K 16514 A, B(\mathrm{OBI})$

MEXICO: Colima: beach $3 \mathrm{mi}$ SE of turnoff to Cuyutlán, KL15132A,B,C,D (OBI); Michoacán: Playa Azul, $K L 15283 A, C$ (OBI); Sinaloa: Playa la Queta N of La Cruz, KL15059A,B (OBI); 9 mi NW of El Dorado, KL15056C, G (OBI)

UNITED STATES: Arizona: Maricopa Co.: I-10 at Maricopa Rd exit, K12546A (OBI)

DOMINICAN REPUBLIC: Distrito Nacional: Santo Domingo, K16424 pop. (OBI); MEXICO: Jalisco: Puerto Vallarta, KL15101A (OBI); 17 mi S of El Tuito, KL15115 pop. (OBI); 4 mi N of Río Tomatlán on Hwy 200, KL15118 pop. (OBI); Ajijic, KL15612A (OBI); México: $5.6 \mathrm{mi}$ $\mathrm{S}$ of Ixtapan de la Sal, KL15434A (OBI); Oaxaca: $12.1 \mathrm{mi}$ SE of Totolapan, KL15541 pop. (OBI); PUERTO RICO: Boqueron, K16513A,B (OBI); Guayama, K16520A (OBI); $12 \mathrm{mi}$ E of Guayama, K16521A (OBI); UNITED STATES: Florida: Dade Co.: along US $4118 \mathrm{mi} \mathrm{W}$ of Florida Turnpike, K16474A (OBI); Collier Co.: $6 \mathrm{mi}$ E of Royal Palm Hammock, K16486A (OBI); Collier Co.: $7 \mathrm{mi}$ NW of Royal Palm Hammock, K16490A (OBI)

MEXICO: Sinaloa: $0.3 \mathrm{mi} \mathrm{W}$ of Las Cascabeles, KL15051 pop. (OBI)

MEXICO: Oaxaca: on Rt 190, $5.8 \mathrm{mi} \mathrm{SE}$ of jct with Rt $125, K L 15494$ pop. (OBI); on Rt 190 , $8.3 \mathrm{mi}$ SE of jct with Rt $125, K L 15497$ pop. (OBI); $0.8 \mathrm{mi}$ SE of turnoff to Mitla on Rt 190, KL15527 pop. (OBI)

MEXICO: Oaxaca: $11.4 \mathrm{mi}$ NE of Juchitlán de Zaragoza, $K L 15556 A, B, C$; Salina Cruz, $K L 15567 A, B$ (OBI)

BOLIVIA: Chuquisaca: Sucre, Canne 2777 (OBI)

ANTIGUA AND BARBUDA: Barbuda: Codrington, K16558A (OBI); along sand rd to Palmetto Pt., K16564A, pop. (OBI); JAMAICA: Hanover 
TABle 1. Continued

\begin{tabular}{|c|c|c|}
\hline $\begin{array}{l}\text { Tribe } \\
\text { Taxon or hybrid combination }\end{array}$ & Chromosome count & Location and voucher \\
\hline & & $\begin{array}{l}\text { Parish: } 3.5 \mathrm{mi} \text { W of Lucea, K16399 pop. (OBI); } \\
6 \mathrm{mi} \text { W of Lucea, K16400A (OBI); } 1.8 \mathrm{mi} \mathrm{SW} \\
\text { of Green Island, K16401 pop. (OBI); St. An- } \\
\text { drew's Parish: Beverley Hills above Kingston, } \\
K B 16411 A(\mathrm{OBI}) \text {; St. James' Parish: Montego } \\
\text { Bay, K16386B,C,D (OBI); DOMINICAN RE- } \\
\text { PUBLIC: La Altagracia Province: Boca de } \\
\text { Yuma, KPr16447A,B,C (OBI) }\end{array}$ \\
\hline $\begin{array}{l}\text { Pectis uniaristata DC. var. jangadensis } \\
\text { (S. Moore) Keil }\end{array}$ & $2 n=12_{11}$ & $\begin{array}{l}\text { MEXICO: Jalisco: } 10.1 \mathrm{mi} \text { E of Magdalena, } \\
\text { KL15635A,B (OBI); Michoacán: } 16.3 \mathrm{mi} \text { S of } \\
\text { Uruapan, } K L 15196 \text { pop. (OBI) }\end{array}$ \\
\hline *Pectis uniaristata DC. var. uniaristata & $2 n=12_{11}$ & $\begin{array}{l}\text { MEXICO: México: } 4 \mathrm{mi} \mathrm{W} \text { of jct with Rt } 134 \text { on } \\
\text { rd to Luvianos, } K L 15427 B, C \text { (OBI); } 3.6 \mathrm{mi} \mathrm{NE} \\
\text { of Tejupilco, KL15429B (OBI); Michoacán: } 8 \\
\text { mi S of Cuatro Caminos, KL15247A,B,D (OBI); } \\
4 \mathrm{mi} \text { N of Pte. El Guayabito on Rt } 37, \\
K L 15276 A \text { (OBI) (bridge + fragment) }\end{array}$ \\
\hline${ }^{*}$ Pectis vollmeri Wiggins & $2 n=12_{\mathrm{Il}}$ & $\begin{array}{l}\text { MEXICO: Baja California Sur: } 9.5 \mathrm{mi} \mathrm{S} \text { of Santa } \\
\text { Rosalía, Keil et al., } 16626 \text { (OBI) }\end{array}$ \\
\hline Porophyllum sp. & $2 n=12_{\mathrm{II}}$ & $\begin{array}{l}\text { MEXICO: Oaxaca: Rt } 190,3.3 \mathrm{mi} \mathrm{SE} \text { of Rt } 125 \\
\text { to Tehuacán, KL15490 pop. (OBI); Puebla: } 12 \\
\text { mi SE of Acatlán, KL15479 (OBI) }\end{array}$ \\
\hline *Tagetes coronopifolia Willd. & $n=12$ & $\begin{array}{l}\text { MEXICO: México: } 5 \mathrm{mi} \text { E of Valle de Brava, } \\
\text { KL15423 pop. (OBI) }\end{array}$ \\
\hline Tagetes lemmonii A. Gray & $2 n=12_{11}$ & $\begin{array}{l}\text { UNITED STATES: Arizona: Santa Cruz Co.: } 10 \\
\text { mi from Patagonia on Harshaw Rd, K12596 } \\
\text { (OBI) }\end{array}$ \\
\hline Tagetes lucida Cav. & $2 n=11_{\mathrm{II}}$ & $\begin{array}{l}\text { MEXICO: Guerrero: Rt } 95,4.8 \mathrm{mi} \mathrm{S} \text { of jct with } \\
\text { Rt } 55, K L 15353 A(\mathrm{OBI})\end{array}$ \\
\hline & $2 n=8_{\mathrm{II}}+3_{\mathrm{III}}$ & $\begin{array}{l}\text { Jalisco: } 18 \mathrm{mi} \text { N of Tamazula, } K L 15143(\mathrm{OBI}) \\
\text { The III's fail to segregate at anaphase I and } \\
\text { form several (up to 6) micronuclei at telo- } \\
\text { phase I }\end{array}$ \\
\hline Tagetes tenuifolia Cav. & $2 n=12_{\mathrm{II}}$ & $\begin{array}{l}\text { MEXICO: México: } 5.6 \mathrm{mi} \mathrm{S} \text { of Ixtapan de Sal on } \\
\text { Rt } 55, K L 15435 \text { pop. (OBI); Oaxaca: } 8 \mathrm{mi} \mathrm{NW} \\
\text { of Tamazulapan on Rt } 190, K L 15502 A(\mathrm{OBI})\end{array}$ \\
\hline \multicolumn{3}{|l|}{ VERNONIEAE } \\
\hline Elephantopus mollis H.B.K. & $2 n=11_{\mathrm{II}}$ & $\begin{array}{l}\text { DOMINICAN REPUBLIC: La Vega Province: } \\
17.5 \mathrm{~km} \text { S of La Vega, } K 16488 A(\mathrm{OBI})\end{array}$ \\
\hline Elephantopus spicatus Juss. ex Aubl. & $2 n=13_{11}$ & $\begin{array}{l}\text { DOMINICAN REPUBLIC: La Altagracia Prov- } \\
\text { ince: Boca de Yuma, K16445 pop. (OBI) }\end{array}$ \\
\hline Vernonia acaulis (Walt.) Gleason & $n=18$ & $\begin{array}{l}\text { UNITED STATES: North Carolina: Cumberland } \\
\text { Co.: Fort Bragg, } K 11652 A(\mathrm{ASU})\end{array}$ \\
\hline Vernonia cinerea (L.) Less. & $2 n=9_{\mathrm{II}}$ & $\begin{array}{l}\text { JAMAICA: Hanover Parish: } 1.8 \mathrm{mi} \mathrm{SW} \text { of Green } \\
\text { Island, } K 16402 A(\mathrm{OBI})\end{array}$ \\
\hline
\end{tabular}

of $E$. bellioides are in accordance with all of these features.

Our reports for Heterotheca accord well with the established base of $x=9$ for the genus (Semple, 1977; Semple, Bloc, and Heiman, 1980). Our count of $2 n=9_{\text {II }}$ for $H$. mucronata is the first report at the diploid level for this taxon; a tetraploid count of $2 n=18_{\text {II }}$ was published in the protologue of this species (Turner, 1984). The count of $2 n=3 x=9_{\text {III }}$ for $H$. villosa $\times H$. fulcrata is the first report for this hybrid combination and probably represents a cross between a tetraploid $H$. villosa (variety not determined) and a diploid $H$. fulcrata. Heterotheca villosa is known at both the diploid and tetraploid levels (Semple, 1977), whereas all reports to date for $H$. fulcrata are diploids. Semple (1977) reported a triploid in the closely related $H$. horrida (Rydb.) Harms.

Cardueae-Cirsium occidentale var. compactum is a rare taxon limited to a narrow coastal strip of San Luis Obispo County, California. Our first report of $2 n=15_{\text {II }}$ for this 
taxon indicates that it is cytologically similar to the more widespread var. occidentale.

Eupatorieae-Our first reports for Brickellia chenopodina $\left(2 n=9_{\mathrm{II}}\right)$ and $2 n=10_{\mathrm{II}}$ for $\mathrm{Ko}$ anophyllon villosum (Eupatorium villosum $\mathrm{Sw}$.) are in accordance with the established base numbers, $x=9$ and $x=10$, respectively, for these genera (King et al., 1976).

Heliantheae-Chromosome counts of $2 n=$ 36 (or $n=18$ ) have been reported previously for Bahia dissecta by several authors. The three species of Bahia most similar to B. dissecta and the closely related genus, Hymenothrix, all have chromosome counts of $n=12$. Ellison (1964), in an attempt to explain the anomalous $n=$ 18 of $B$. dissecta, speculated that Bahia has a base of $x=4$, and that Bahia dissecta somehow originated by hybridization between a triploid $(3 x=12)$ and a hexaploid $(6 x=24)$. However, neither the hypothesized triploid nor any diploid $(2 x=8)$ species based on the putative $x=4$ are known in Bahia. We believe that a much simpler explanation for the 36 chromosomes of $B$. dissecta is that this species is a stabilized apomictic triploid $(3 n=3 x=36)$. A base of $x=12$ is supported by counts of the close relatives of $B$. dissecta. Apomixis is supported by the fact that despite only a small amount of sporogenous tissue in the anthers [sometimes it is entirely absent!], abnormal diad pollen, and very irregular meiosis, $B$. dissecta, nonetheless, has a high seed set. Strother (1977) noted a similar phenomenon in triploid populations of Thymophylla [Dyssodia] tenuiloba.

Previously reported chromosome counts for Chrysanthellum support a base of $x=8$ (Turner et al., 1979). Our report of $2 n=9_{\text {II }}$ for $C$. michoacanum indicates either that dysploidy has been a factor in the evolution of the genus or the presence of a supernumary bivalent.

Cosmos caudatus has been reported previously as a tetraploid $(n=24)$, by Turner and Irwin (1960), Turner and King (1964), and Melchert (1968). McVaugh (1984) noted that although this species commonly has ray ligules $1-1.5 \mathrm{~cm}$ long, plants from the Nueva Galicia region of westcentral Mexico (where our vouchers were collected) commonly have ligules $2-3 \mathrm{~cm}$ long. The diploid $(n=12)$ condition of the long-rayed plant is an indication that these plants should be further investigated to determine whether they are taxonomically distinct from the short-rayed tetraploids.

Powell (1978) reported that chromosome counts are known from all but three species of
Flaveria. Our first report of $n=18$ for $F$. angustifolia accounts for one of the remaining species. This count is in accord with the base of $x=18$ for the genus.

Previously reported chromosome counts have indicated that Florestina is dibasic with $x=10$ and 12 (Turner, 1963). Turner considered $F$. latifolia (DC.) Rydb. $(n=12)$ and $F$. platyphylla to be very closely related on morphological grounds. Our first report for $F$. platyphylla $(n=12)$ supports this relationship. All previous reports for other species of Florestina have been $n=10$. Our count of $n=11$ from a single population of $F$. pedata is the first report of this chromosome number for the genus.

Galinsoga parviflora var. parviflora, a widespread taxon, is apparently a diploid $(n=8)$ throughout its range. Canne (1983) reported a tetraploid count $(n=16)$ for a collection of Galinsoga parviflora var. semicalva from northwestern Chihuahua. Our report for var. semicalva (also $n=16$ ) is from southeastern Arizona ca. $300 \mathrm{~km}$ to the northwest. This evidence suggests that the count reported by Canne is not an isolated occurrence and that the tetraploids may have a significant range.

Our count of $2 n=12_{\text {II }}$ for Hymenothrix palmeri, the first report for this wholly Mexican species, accords well with the base of $x=12$ established for species occurring in the United States. This species appears to be geographically isolated from the hybrid complex involving $H$. wislizenii A. Gray $(n=12), H$. wrightii A. Gray $(n=12)$, and $H$. loomisii S. F. Blake $(n=24)$ currently under investigation (Clark, Keil, and Pinkava, 1978; Pinkava and Keil, 1986).

Chromosome counts of $n=11,12,14$, and 18 have been reported previously for species of Sclerocarpus (Robinson et al., 1981). Our count of $2 n=13_{\text {II }}$ for $S$. papposus is the first report of this number for the genus. Sclerocarpus was placed by Stuessy (1977) in subtribe Verbesininae in which the majority of genera have base numbers of $x=15,16$ or 17 . Robinson (1981) placed Sclerocarpus in the Helianthinae (defined differently than by Stuessy) in which the majority of genera have a base of $x=17$. If either placement is correct, the dysploid series of chromosome numbers in Sclerocarpus is apparently reductional.

Interspecific hybridization is well-known in Silphium $(x=7)$ both in the field and under experimental conditions (Fisher, 1959; Settle and Fisher, 1972). The count reported here $\left(2 n=7_{\text {II }}+1_{\mathrm{I}}\right)$ probably represents a secondgeneration hybrid or backcross. At anaphase I 
the univalent either lags at the metaphase plane and at telophase I forms a micronucleus, or is incorporated into one of the principal daughter nuclei. The very low pollen stainability and the presence of micro- and macropollen indicate that this plant is highly sterile.

Stuessya, a genus of three species, was described by Turner and Davies (1980) from southcentral Mexico. They reported that Stuessya perennans has a chromosome number of $n=17$. Our reports of $n=17$ for both $S$. perennans and for $S$. michoacana support $x=$ 17 as the base for this genus. McVaugh (1984) did not recognize Stuessya as distinct from $\mathrm{Vi}$ guiera, which also has a base of $x=17$. Further research on the systematics of Viguiera will be required to determine whether Steussya is worthy of recognition.

The count of $2 n=22_{\text {II }}$ for Wedelia sp. (Keil and Luckow 15201) represents an apparently undescribed taxon (J. Strother, personal communication). Wedelia is a cytologically complex genus with an apparent aneuploid series of base numbers ranging from $x=10$ to $x=$ 15 and a complicated combination of polyploid and further aneuploid changes. There is considerable doubt as to the precise circumscription of the genus (McVaugh, 1984). The questions of whether Wedelia, as traditionally defined, is a monophyletic taxon and what its relationship is to Aspilia and some other helianthoid genera are being investigated ( $\mathrm{J}$. Strother, personal communication).

Inuleae-Our first report $\left(2 n=14_{I I}\right)$ for $G n a$ phalium californicum indicates that this species, like many other native North American members of the genus, is a tetraploid, on the wellestablished base of $x=7$ for subtribe Gnaphalinae (Merxmüller et al., 1977).

Lactuceae-Tragopogon mirus and T. miscellus Owenbey, spontaneous amphidiploid hybrids derived from Eurasian parental taxa in western North America (Owenbey, 1950) were both reported to occur in Arizona by Brown and Schaack (1972). A biosystematic investigation of the Arizona species of Tragopogon (Brown, 1974) resulted in a reinterpretation of the findings of Brown and Schaack. Capitula of $F_{1}$ generation, allotetraploid Tragopogon mirus (T. dubius Scop. $\times T$. porrifolius L.) exhibit bicolored ligules (distally pale to dark violet-purple and proximally pale lemon-yellow), similar to the corresponding, somewhat sterile diploid hybrids (percent pollen stainability $=48.7 \pm 15.0, \mathrm{~N}=8$ individuals), but with much larger heads and a greater number of florets (Brown, 1974). In $F_{2}$ and subsequent generations of $T$. mirus, ligule coloration is variable, sometimes with all completely pale lemon-yellow ligules as in Brown 390-6 and Schaack 479 (tentatively reported as T. miscellus by Brown and Schaack, 1972). However, this yellow-flowered form of $T$. $m i$ rus differs from $T$. miscellus ( $T$. dubius $\times T$. pratensis L., $4 x$ ) by lacking the effects of the genome of $T$. pratensis (and also $T$. miscellus), namely leaf apices strongly reflexed and curled (not slightly deflexed or straight as in T. mirus), leaf bases well crisped (not flat), bases of phyllaries with purple margins (not with green or greenish yellow margins), and the $T$. pratensis flavonoid profile (Brown, 1974). Furthermore, $T$. pratensis is rare in Arizona and very distant from the Flagstaff population of $T$. mirus. Tragopogon miscellus is not known from Arizona.

Tageteae-Counts of $n=12,24$, and 36 for various species of Pectis support the well-established base of $x=12$ for this genus (Keil, 1977). Over half of the species of Pectis have now been counted. Euploid levels that have been documented include $2 x, 3 x, 4 x, 6 x$, and $8 x$ (Keil, 1977, 1984). Except for the presence of a variable number of small supernumerary chromosomes in a few individuals of Pectis angustifolia Torr., $P$. papposa, and their hybrids (Keil, 1977), dysploid changes have not been documented in this genus.

The proportion of polyploid species of Pectis in the West Indies and peninsular Florida is apparently considerably higher than in the mainland areas of North America. This is possibly the result of dispersal of facultatively autogamous species from mainland areas to insular locations followed by hybridization and allopolyploidy. The ability of various species in the West Indies and Florida to hybridize in nature is documented here by three different hybrid combinations. Retention of facultative autogamy in polyploidized hybrids would allow them to establish insular populations without being swamped by gene flow from established parental taxa.

Pectis latisquama was previously reported by Powell and Turner (1963) to be a tetraploid $(n=$ ca. 24). Our counts of $2 n=72$ indicate that this species has a hexaploid race as well. No morphological characters have been discovered that distinguish the tetraploids (as represented by King 3557, the voucher for Powell and Turner's report) and the hexaploids. Another perennial species, Pectis longipes A. Gray, has two chromosomal races which are also morphologically very similar (Luckow, 1983) but the races differ in the odor of their 
essential oils. Unlike $P$. longipes, however, $P$. latisquama lacks scented oils.

The sterile triploid hybrid, Pectis $\times$ floridana between Pectis prostrata $(2 x)$ and $P$. glaucescens $(4 x)$ is morphologically very similar to $P$. linearifolia $(4 x)$, and hybrid individuals have been misidentified as the latter taxon (Keil, 1987). The triploid hybrid and $P$. linearifolia differ, however, in several minor morphological features, and it is unlikely that this resemblance is more than coincidental.

The undetermined species of Porophyllum for which we are reporting a count of $2 n=12_{\text {II }}$ is apparently a part of the complex of cultivated and wild species of this genus discussed by Bretting and Hernández (1982). These plants do not correspond precisely with any of the species included by Johnson (1969) in his treatment of Porophyllum. They most closely resemble $P$. filifolium A. Gray but are annual instead of perennial. The ethnobotanical study of Bretting and Hernández indicates that the species of this genus in southern Mexico are in need of further taxonomic investigation.

\section{LITERATURE CITED}

BEEKS, R. M. 1955. Improvements in the squash technique for plant chromosomes. Aliso 3: 131-134.

Bretting, P. K., ANd E. Hernández X. 1982. Papaloquelite y la etnobotanica de las especies de Porophyllum en México. Biotica 7: 191-203.

Brown, R. C., AND C. G. SchaAck. 1972. Two new species Tragopogon for Arizona. Madroño 21: 304.

Brown, R. K. 1974. Biosystematics of Tragopogon (Compositae: Cichorieae) in Arizona. M.Sc. thesis, Arizona State University, Tempe.

CANNE, J. 1983. Cytological and morphological obser- vations in Galinsoga and related genera (Asteraceae). Rhodora 85: 355-366.

Clark, W. D., D. J. Keil, and D. J. Pinkava. 1978. Cytological and chemical evidence for the origin of Hymenothrix loomisii Blake (Compositae). Bot. Soc. Amer. Misc. Publ. 156: 50.

$\rightarrow$ Cronguist, A. 1943. The separation of Erigeron from Conyza. Bull. Torrey Bot. Club 70: 629-632.

Ellison, W. L. 1964. A systematic study of the genus Bahia (Compositae). Rhodora 66: 69-86, 177-215, 293-311.

FISHER, T. R. 1959. Natural hybridization between Silphium laciniatum and Silphium terebinthinaceum. Brittonia 11: 250-254.

JoHnson, R. R. 1969. Monograph of the plant genus Porophyllum. Univ. Kansas Sci. Bull. 48: 225-267.

KeIL, D. J. 1977. Chromosome studies in North and Central American species of Pectis L. (Compositae: Tageteae). Rhodora 79: 79-94.

-1984. New species of Pectis (Asteraceae) from the West Indies, Mexico, and South America. Brittonia 36: 74-80.

- 1987. Synopsis of the Florida species of Pectis (Asteraceae). Sida 11: 385-395.

$\rightarrow-$, AND D. J. Pinkava. 1976. Chromosome counts and taxonomic notes for Compositae from the United States and Mexico. Amer. J. Bot. 63: 1393-1403.
一 AND - 1979. In A. Löve [ed.], IOPB Chromosome number reports LXIII, 271-273. Taxon 28: 265-279.

— mosome number reports LXXII, 705-706. Taxon 30: 694-708.

- AND T. F. StUESSY. 1975. Chromosome counts of Compositae from the United States, Mexico and Guatemala. Rhodora 77: 171-195.

$\longrightarrow \rightarrow-$, AND $\longrightarrow$ 1977. Chromosome counts of Compositae from Mexico and the United States. Amer. J. Bot. 64: 791-798.

King, R. M., D. W. Kyhos, A. M. Powell, P. H. Raven, AND H. Robinson. 1976 (1977). Chromosome numbers in Compositae, XIII. Eupatorieae. Ann. Missouri Bot. Gard. 63: 862-888.

LuCKow, M. 1983. A morphometric and cytological study of Pectis longipes A. Gray (Compositae: Tageteae). M. Sc. thesis, California Polytechnic State University, San Luis Obispo.

MCVAugh, R. 1984. Flora Novo-Galiciana, Vol. 12, Compositae. University of Michigan Press, Ann Arbor.

Melchert, T. E. 1968. Systematic studies in the Coreopsidinae: cytotaxonomy of Mexican and Guatemalan Cosmos. Amer. J. Bot. 55: 345-353.

MerXmüller, H., P. LeINS, AND H. Roessler. 1977. Inuleae-systematic review. In V. H. Heywood, J. B. Harborne, and B. L. Turner [eds.], The biology and chemistry of the Compositae, 577-602. Academic Press, London and New York.

OWENBEY, M. 1950. Natural hybridization and amphiploidy in the genus Tragopogon. Amer. J. Bot. 37: 487-499.

Pinkava, D. J., AND D. J. KeIl. 1977. Chromosome counts of Compositae from the United States and Mexico. Amer. J. Bot. 64: 680-686.

$\longrightarrow$ AND -1986 . On the origin and success of an Arizona endemic amphidiploid, Hymenothrix loomisii Blake (Compositae). J. Ariz. Nev. Acad. Sci. 21 (Proc. Suppl.): 10.

Powell, A. M. 1978. Systematics of Flaveria (Flaveriinae-Asteraceae). Ann. Missouri Bot. Gard. 65: 590636.

, AND B. L. TURNER. 1963. Chromosome numbers in the Compositae. VII. Additional species from the southwestern United States and Mexico. Madroño 17: 128-140.

Robinson, H. 1981. A revision of the tribal and subtribal limits of the Heliantheae (Asteraceae). Smithsonian Contr. Bot. 51: 1-102.

- A. M. Powell, R. M. KING, AND J. F. Weedin. 1981. Chromosome numbers in Compositae, XII: Heliantheae. Smithsonian Contr. Bot. 52: 1-28.

Semple, J. C. 1977. Cytotaxonomy of Chrysopsis and Heterotheca (Compositae-Astereae): a new interpretation of phylogeny. Canad. J. Bot. 55: 2503-2513.

- , V. C. Bloc, AND P. Heiman. 1980. Morphological, anatomical, habit, and habitat differences among the goldenaster genera Chrysopsis, Heterotheca, and Pityopsis (Compositae: Astereae). Canad. J. Bot. 58: 147-163.

Settle, W., AND T. R. Fisher. 1972. A biosystematic study of Silphium integrifolium Michaux (Compositae). Ohio J. Sci. 72: 254-265.

SNow, R. 1963. Alcoholic hydrochloric acid-carmine as a stain for chromosomes in squash preparations. Stain Tech. 38: 9-13.

Stuessy, T. F. 1977. Heliantheae-systematic review. 
In V. H. Heywood, J. B. Harborne, and B. L. Turner [eds.], The biology and chemistry of the Compositae, 621-671. Academic Press, London and New York.

Strother, J. L. 1977. Tageteae-systematic review. In V. H. Heywood, J. B. Harborne, and B. L. Turner [eds.], The biology and chemistry of the Compositae, 769-783. Academic Press, London and New York.

TURNER, B. L. 1963. Taxonomy of Florestina (Helenieae, Compositae). Brittonia 15:27-46.

- 1984. Three new species of Heterotheca (Asteraceae-Astereae) from northern Mexico. Phytologia 55: 204-207.

$\rightarrow-$, J. Bacon, L. UrbatsCh, ANd B. Simpson. 1979.
Chromosome numbers in South American Compositae. Amer. J. Bot. 66: 173-178.

, AND F. DAvies. 1980. Stuessya (Asteraceae: Heliantheae), a new genus from southcentral Mexico. Brittonia 32: 209-212.

, AND H. S. IRwIN. 1960. Chromosome numbers in the Compositae. II. Meiotic counts for fourteen species of Brazilian Compositae. Rhodora 662: 122126.

$\longrightarrow \rightarrow-$ AND R. M. KING. 1964. Chromosome numbers in the Compositae. VIII. Mexican and Central American species. Southw. Naturalist 9: 27-39. 